\title{
An Experiment to Test Advanced Materials Impacted by Intense Proton Pulses at CERN HiRadMat Facility
}

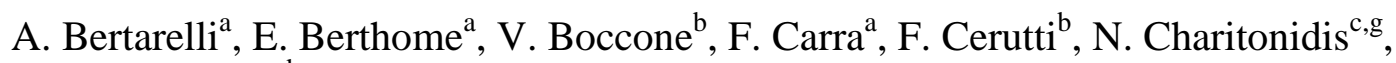 \\ C. Charrondiere ${ }^{\text {d }}$, A. Dallocchio ${ }^{\text {a }}$, P. Fernandez Carmona ${ }^{a}$, P. Francon ${ }^{\text {a }}$, L. Gentini ${ }^{\text {a }}$, \\ M. Guinchard ${ }^{\mathrm{a}}$, N. Mariani ${ }^{\mathrm{a}}$, A. Masi ${ }^{\mathrm{b}}$, S. D. Marques dos Santos ${ }^{\mathrm{a}}$, P. Moyret $^{\mathrm{a}}$, L. Peroni $^{\mathrm{f}}$, \\ S. Redaelli ${ }^{\mathrm{e}}$ M. Scapin ${ }^{\mathrm{f}}$ \\ ${ }^{a}$ CERN, EN-MME, CH-1211 Geneva 23, Switzerland \\ ${ }^{\mathrm{b}}$ CERN, EN-STI, CH-1211 Geneva 23, Switzerland \\ ${ }^{c}$ CERN, EN-MEF, CH-1211 Geneva 23, Switzerland \\ ${ }^{\mathrm{d}}$ CERN, EN-ICE, CH-1211 Geneva 23, Switzerland \\ ${ }^{\mathrm{e}}$ CERN, BE-ABP, CH-1211 Geneva 23, Switzerland \\ ${ }^{\mathrm{f}}$ Politecnico di Torino, I-10129 Torino, Italy \\ ${ }^{\mathrm{g}}$ École Polytechnique Fédéral de Lausanne (EPFL), CH-1015, Lausanne, Switzerland
}

Keywords: HiRadMat, LHC Collimation Upgrade, High Power Target Systems, Beam Intercepting Devices, Shock Waves, Hydrocodes.

\section{Summary}

Predicting the consequences of highly energetic particle beams impacting protection devices as collimators or high power target stations is a fundamental issue in the design of state-ofthe-art facilities for high-energy particle physics.

These complex dynamic phenomena, which may induce material phase transitions, extended density changes, shock waves generation, explosions, material fragment projections etc., have been successfully simulated resorting to highly non-linear numerical tools (Hydrocodes).

In order to produce accurate results, however, these codes require reliable material constitutive models that, at the extreme conditions induced by a destructive beam impact, are scarce and often inaccurate.

In order to derive or validate such models (Equations of State, Strength Models, Failure Models), a comprehensive, first-of-its-kind experiment has been recently carried out at CERN HiRadMat facility: performed tests entailed the controlled impact of intense and energetic proton pulses on a number of specimens made of six different materials.

Experimental data were acquired, mostly in real time, relying on extensive embedded instrumentation (strain gauges, temperature and vacuum sensors) and on remote acquisition devices (laser Doppler vibrometer and high-speed camera). The dynamic ranges of the digital acquisition system were sufficient to acquire the very fast and intense shock waves generated by the impact. The high speed video camera allowed capturing a number of frames during the time of flight of the material fragments projected away from impacted specimens. This information is then benchmarked against advanced numerical simulations.

Preliminary results of the tests are discussed. In depth post-irradiation analyses are foreseen once the specimens have reached a sufficiently low level of activation.

The experimental method presented in this paper may find applications to test materials under very high strain rates and temperatures in domains well beyond particle physics (severe accidents in fusion and fission nuclear facilities, space debris impacts, fast and intense loadings on materials and structures etc.). 


\section{Contents}

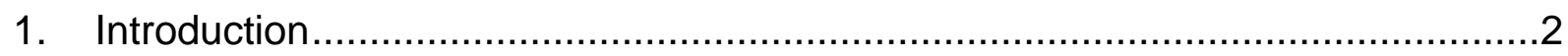

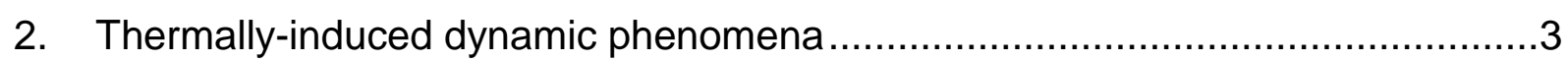

3. Material modelling and advanced simulation tools ..........................................

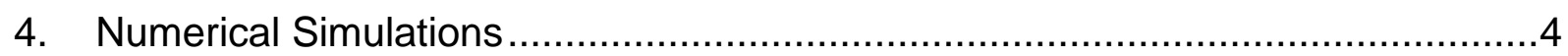

4.1. Transport code / Hydrodynamic code uncoupled solution ............................5

4.2. Transport code / Hydrodynamic code coupled solution .............................

5. The HRMT-14 Experiment at CERN HiRadMat Facility ....................................

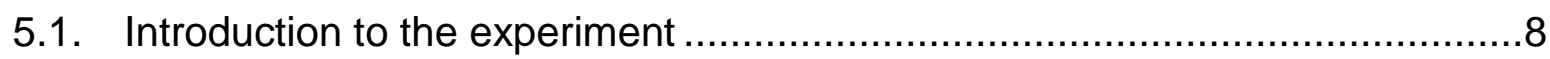

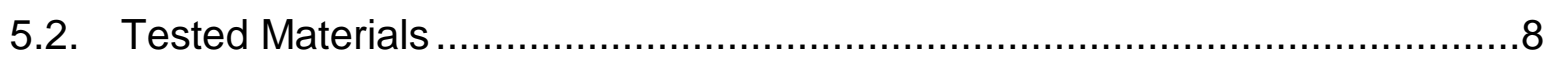

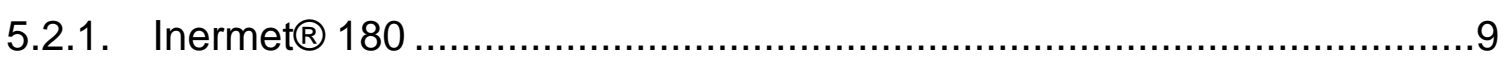

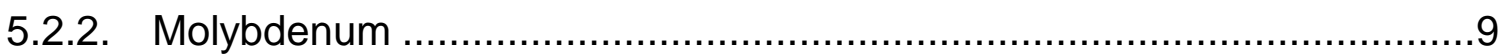

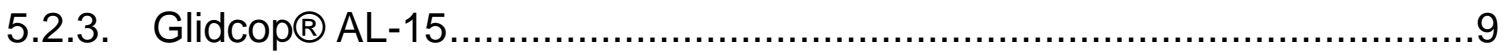

5.2.4. Molybdenum-Copper-Diamond (MoCuCD) ......................................10

5.2.5. Copper-Diamond (CuCD) ......................................................... 10

5.2.6. Molybdenum-Graphite composites (MoGR) .....................................10

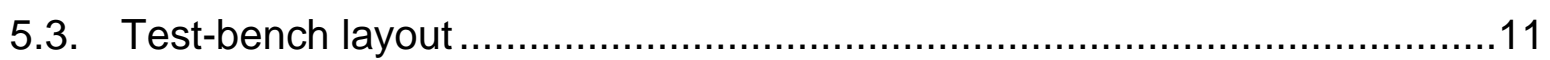

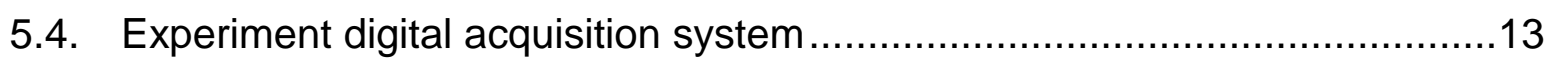

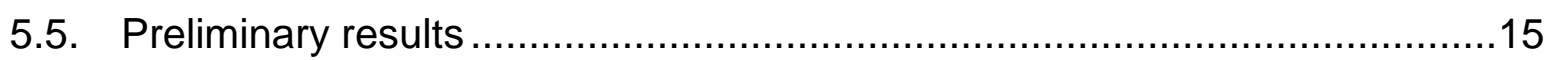

5.6. Discussion of preliminary results and future steps..................................18

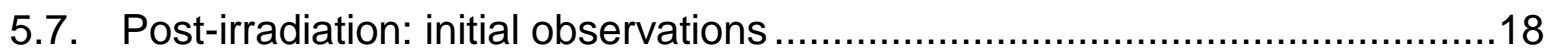

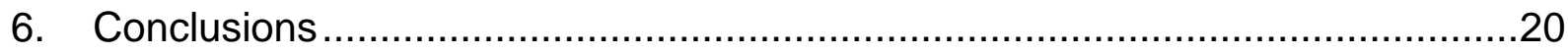

\section{Introduction}

The introduction in recent years of new, extremely energetic particle accelerators such as the Large Hadron Collider (LHC) [1] brought about the need for advanced cleaning and protection systems in order to safely increase the energy and intensity of particle beams to unprecedented levels [2].

A key element of the cleaning and protection system is constituted by collimators (Fig. 1) [3], which are designed to intercept and absorb the intense particle losses unavoidably induced in accelerators and to shield other components from the catastrophic consequences of beam orbit errors [4].

Furthermore, recent ambitious programs for the development of accelerator facilities, aimed at the massive production of elusive particles such as neutrinos or muons, rely on target systems submitted to the impact of proton beams at extraordinary intensities (impact power up to $5 \mathrm{MW}$ ) [5]. Therefore, it is paramount to assess the responses to such potentially destructive events of materials presently used, or being developed for future use, in beam intercepting devices (collimators, targets, dumps, absorbers, spoilers, windows etc.).

Complex numerical methods have been used to study the dynamic phenomena, such as phase transitions, density changes, generation and propagation of shock waves, explosions, 
fragment projections, generated in matter when it is impacted by beams made of highly energetic particles. Unfortunately, the material models which are required to perform such simulations, at the extreme conditions, as to temperature, pressure and density, induced by such impacts, are hardly available in scientific literature; besides, most of the existing information is often classified as it is drawn from military research mainly related to nuclear weaponry. Finally, very little data can be found for non-conventional alloys and compounds.

The experiment presented in the following sections has been devised precisely to bridge this knowledge gap and gather experimental data upon which building and/or validating reliable material constitutive models.

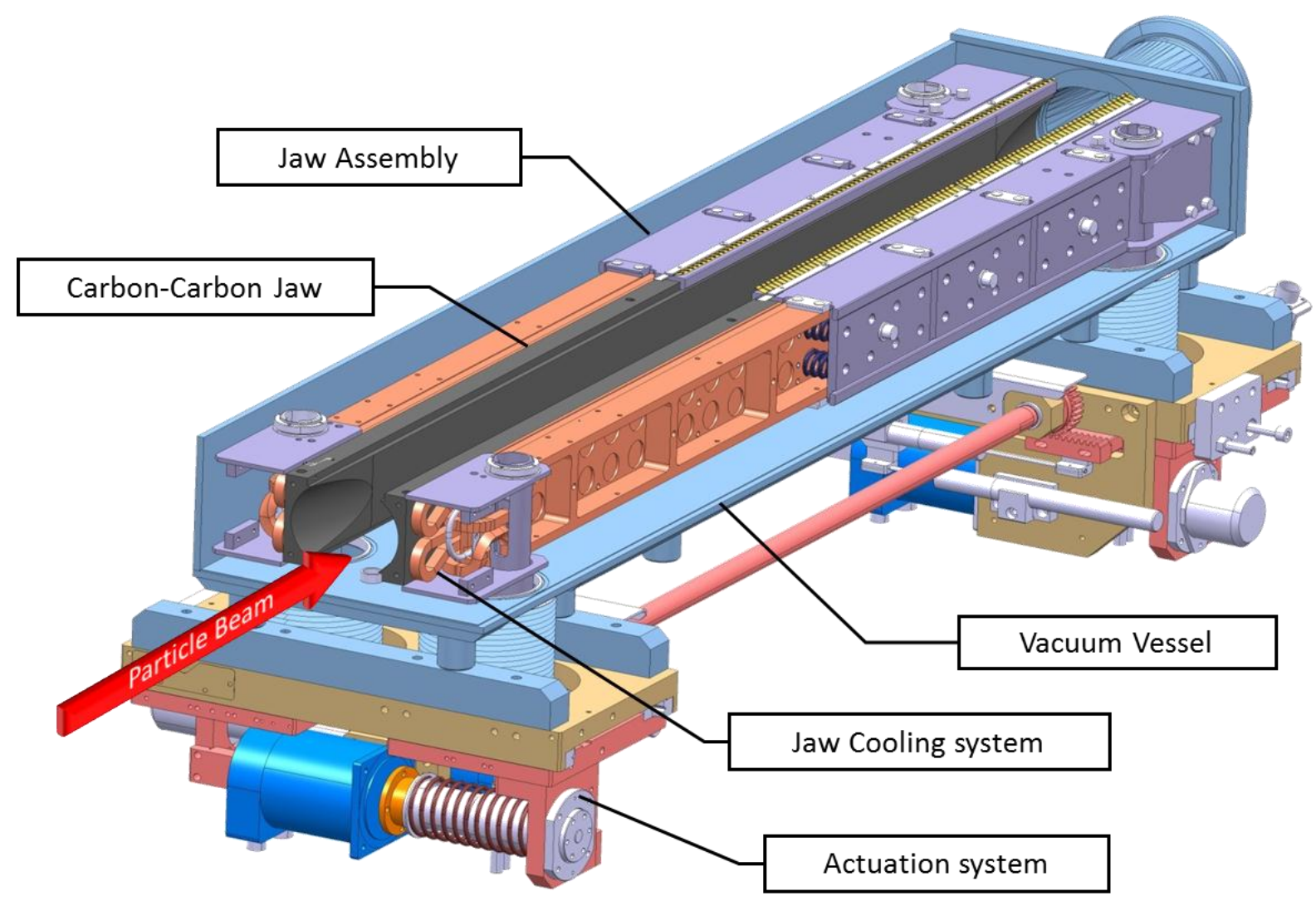

Figure 1. Cutaway of a LHC (Secondary) Collimator

\section{Thermally-induced dynamic phenomena}

It is well known that the rapid interaction of highly energetic particle beams with matter induces dynamic responses in the impacted structure [6], [7]. The nature, intensity and time scale of these responses depend on several parameters, mainly deposited energy and energy density, interaction duration, physical and mechanical properties of the impacted material.

Three dynamic regimes can be identified at increasing deposited energy, namely elastic stress waves, plastic stress waves and shock waves [8].

Elastic stress waves are encountered in cases of relatively low energetic impacts, when induced dynamic stresses do not exceed the material yield strength. Changes of density are negligible and pressure waves propagate at the elastic sound speed $\left(\mathrm{C}_{0}\right)$ without plastic deformation. These phenomena can be effectively treated with standard implicit FEM codes [9] or, in some cases, even with analytical methods [10].

Once the dynamic stresses exceed the material yield strength, plastic stress waves appear propagating at velocities slower than the elastic sound speed $\left(\mathrm{C}<\mathrm{C}_{0}\right)$. Changes of density can still be considered negligible. This regime can be treated, at an acceptable degree of approximation, with standard implicit FEM codes [11]. 
When the deposited energy is high enough to increase strains and stresses over a critical threshold for stresses and strains, a shock wave is formed propagating at a velocity higher than $\mathrm{C}_{0}$, potentially leading to severe damages in the affected component. A shock wave is characterized by a sharp discontinuity in pressure, density and temperature across its front.

\section{Material modelling and advanced simulation tools}

When dealing with changes of phase and significant changes of density, one has to resort to a new class of wave propagation codes, known as hydrocodes. These are highly non-linear finite element tools, using explicit time integration schemes, developed to study very fast and intense loadings on materials and structures [12]. Hydrocodes take their name from the original assumption of pure fluid-like behaviour of the impacted solids, which is typically acceptable when achieved stresses greatly exceed the flow strength of the material and the stress tensor can be reduced to its hydrostatic component only; nowadays the deviatoric behaviour (responsible for material strength) is also taken into account; however the original name is still widely used.

Hydrocodes usually rely on complex material constitutive models encompassing a very large range of densities and temperatures, which, unlike implicit FEM codes, may include large changes of density and phase transitions. Strength and failure models are also more complicated as they have to account for the effects of strain rate, temperature, changes of density etc.

Equations of State (EOS) are integrated in the hydrocode to model the behaviour of materials under any state and condition. They typically provide the evolution of pressure as a function of density, temperature and energy. Most widely adopted analytical EOS are Shock, Tillotson [13] and Mie-Gruneisen; however their application is limited since analytical modelling can describe only a single phase region of the EOS. A tabular EOS, such as SESAME [14], can be employed to appreciate material behaviour over different phases without loss in precision. Additionally, polynomial EOS can be interpolated from tabular data.

To model the behaviour of materials in the extreme conditions due to shock wave propagation, an advanced yielding criterion is needed. The model must take into account, in addition to strain, the strain rate (which in case of shock waves can be as high as $10^{6} \mathrm{~s}^{-1}$ ) and the temperature (above melting point the material practically loses its shear strength and behaves as a fluid). Most used models are Johnson-Cook [14], Steinberg-Guinan [16] and Johnson-Holmquist [17].

On the same basis, dynamic failure models must consider many factors such as strain, strain rate, temperature, maximum and minimum pressure, fracture toughness, etc. In addition, failure criteria also depend on the type of failure and on the mesh used for the simulation. Examples are Maximum Plastic Strain failure criterion, Minimum Hydrostatic Pressure failure criterion and Grady spall model [18].

\section{Numerical Simulations}

A considerable experience in computation of materials and structures under extreme conditions, using state-of-the-art hydrodynamic codes (mainly Autodyn ${ }^{\circledR}[19]$ and LS-Dyna ${ }^{\circledR}$ [20]), has been gathered in recent years at CERN and Politecnico di Torino, partly in the frame of the European Collaboration for Accelerator Research and Development (EuCARD) [21].

These numerical methods are extensively adopted in the design and analysis of complex devices: 3D models routinely encompass several materials and components, with complicated geometries and involve numerical complications like unilateral contacts, interface between parts modeled with different algorithms, impacts, compenetration etc.

The energy deposition maps which are required to perform these studies are computed by Monte-Carlo particle transport codes such as FLUKA [22],[23]. The deposited energy distribution depends on the density of the impacted material; hence a coupling between the transport code and the hydrocode may be necessary if the density significantly changes during 
the time of impact. In the following sections two examples are provided, with and without code coupling.

\subsection{Transport code / Hydrodynamic code uncoupled solution}

The simulation aimed at assessing the consequences of the accidental impacts of proton pulses on the jaw of a Tertiary Collimator for the LHC (Fig. 2) [24]. The core of the collimator jaw is composed of five blocks made of Inermet ${ }^{\circledR} 180$, a commercial tungsten heavy alloy.

The impinging proton pulses are constituted by trains of bunches of $1.3 \times 10^{11}$ particles each with energy up to $5 \mathrm{TeV}$, spaced by $25 \mathrm{~ns}$. Several accident cases, with different degrees of intensity (up to 8 bunches) were simulated; energy deposition maps were obtained from FLUKA. Two complementary 3D models were implemented in Autodyn: 1) a Lagrangian model of the whole collimator jaw and 2) a shorter model of a portion of the collimator, modelled by the Smoothed Particle Hydrodynamics technique (SPH) [25], to simulate the high-speed ejection of particles towards the surrounding structures.

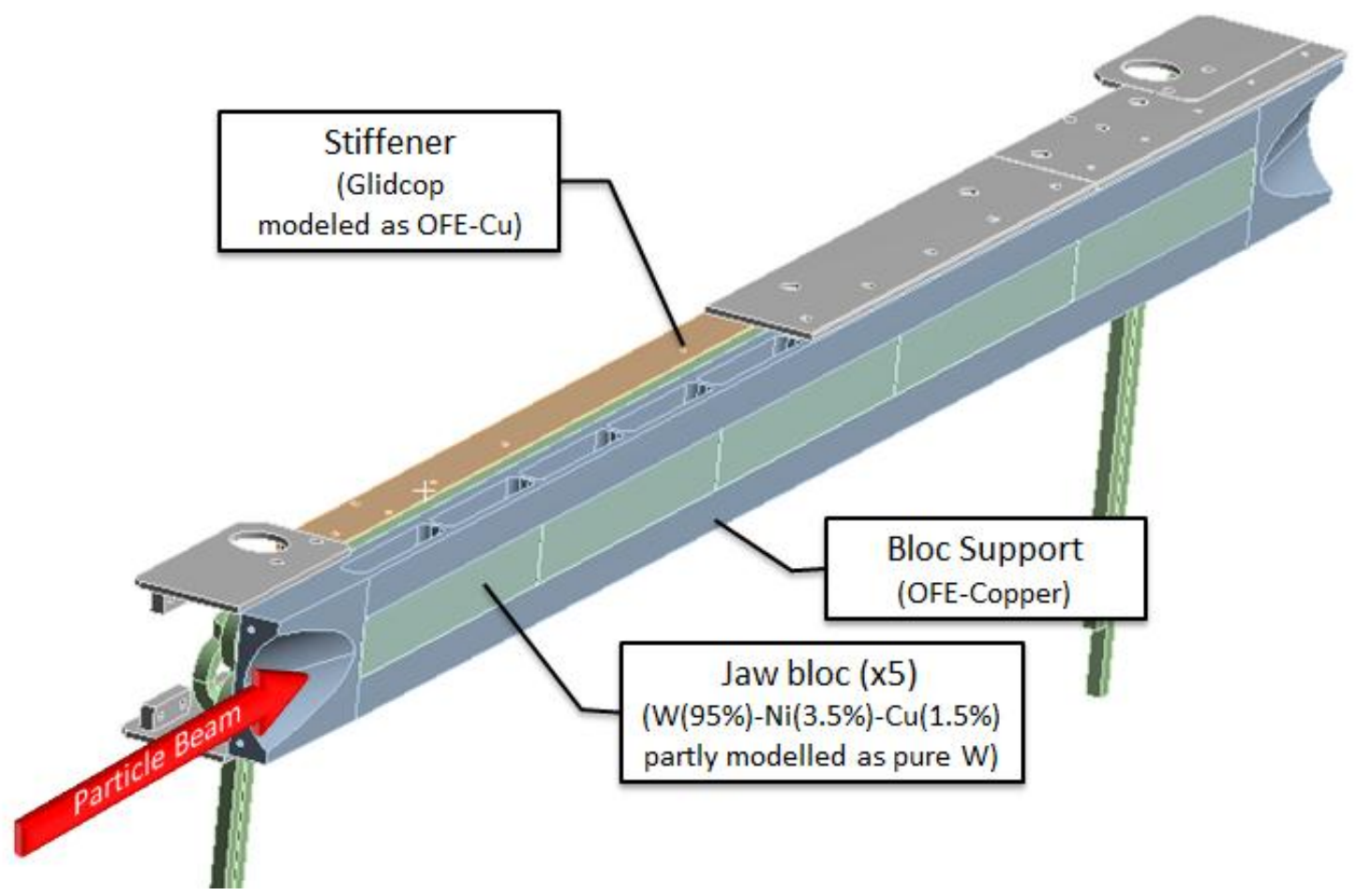

Figure 2. Jaw assembly of a LHC Tertiary collimator

The impact of every particle bunch with matter provokes a sudden increase in temperature and pressure, which in turn generates an outbound shock wave. The rarefaction wave that follows may in turn lead to a substantial reduction in density; hence, one should in principle update the FLUKA model for each bunch and re-perform the simulation with the new density map, as changes in density substantially affect the deposited energy distribution. However, for the simulated accident cases, the change of density during the impact of the bunch train was found negligible: therefore the energy distribution map calculated in the initial state could be used throughout the whole simulation.

It was also found that a key role in determining the damage extension induced by beam impacts on a composite structure is played by the shock impedance matching between adjoining components. Shock impedance in a given material is defined as [26]:

$$
Z=\rho_{0} U_{s}
$$


where $\rho_{0}$ is the initial density and $U_{s}$ is the shock velocity. Due to the large impedance mismatch between tungsten and copper (high $Z_{W} / Z_{C u}$ ratio), most of the wave energy is confined inside the Inermet blocks: this limits the damage produced in other critical components such as the cooling pipes (Fig. 3).

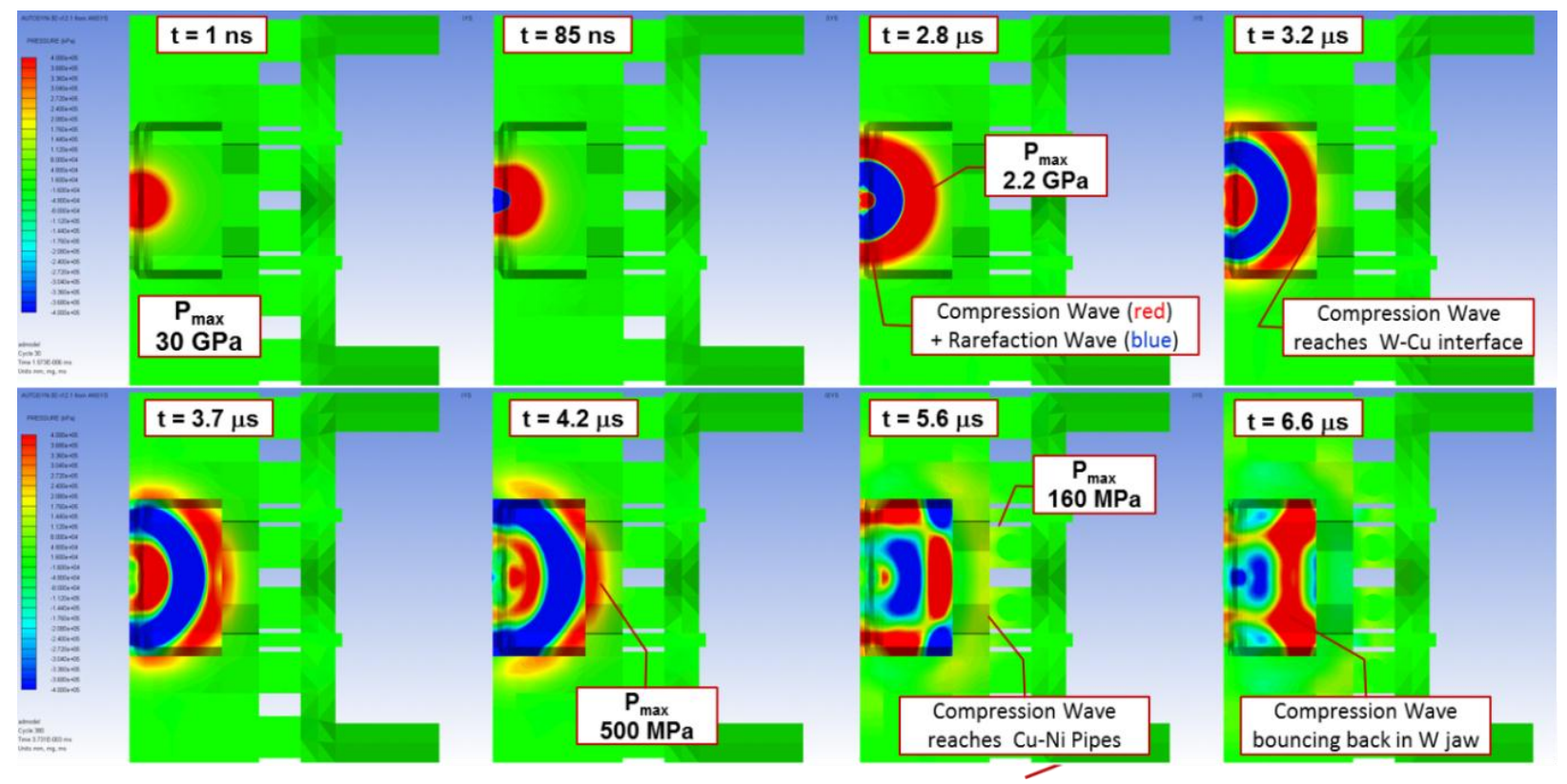

Figure 3. One LHC bunch, $5 \mathrm{TeV}$ : propagation of the shock wave in the jaw assembly

The primary factor determining the extent of damage to the jaw is the total deposited energy. Even in the less destructive cases, a sizeable plastic deformation is found on the copper support and on the cooling circuit; a groove on the surface of impacted Inermet blocks, which can be reproduced with the SPH technique, is also generated while Inermet fragments are projected towards the opposite jaw (Fig. 4).
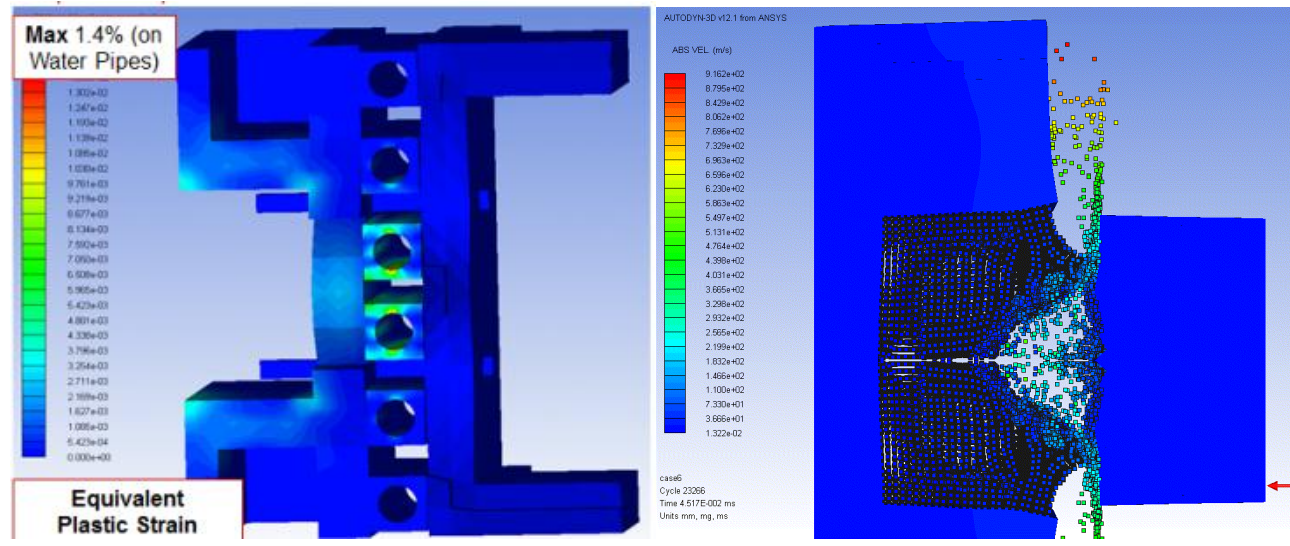

Figure 4. Eight LHC bunches, $5 \mathrm{TeV}$ : W particles projected towards opposite jaw, initial velocity is $\sim 1 \mathrm{~km} / \mathrm{s}$ (right).

An experiment was performed in August 2012 at CERN HiRadMat facility [27] in order to assess the damage extensions in case of accidental beam impact on a LHC tertiary collimator and to benchmark these simulations: the two jaws of a complete collimator were impacted by proton beams at different intensities [28]. Direct observations on the induced damage will be possible in early 2013. 


\subsection{Transport code / Hydrodynamic code coupled solution}

In the previous example the energy deposition calculated for the first bunch on the pristine material was maintained also for subsequent bunches since the change of density induced by the impinging particles was found to be negligible for the duration of the impact. The same approach was followed for similar calculations on other structures [29]. As discussed above, however, the rarefaction wave following the compression shock wave generated by prolonged intense impacts may significantly reduce material density. If subsequent bunches arrive after a lapse of time sufficiently long to allow the rarefaction wave to develop, particles will experience a considerable increase of interaction length and penetrate deeper in matter due to density reduction which, along with temperature rapid augmentation, may also lead to changes of phase, plasma generation and formation of cavities in the core of the material.

A procedure, which takes into account the change of density through iterative coupling between LS-DYNA and FLUKA codes, has recently been developed [30]. The case presented is that of a tungsten cylindrical target impacted at its centre by $30 \mathrm{LHC}$ bunches at the energy of $7 \mathrm{TeV}$.

Results of the first FLUKA simulation, performed on pristine material, are uploaded in the LS-DYNA mechanical model. Then, for each bunch, the algorithm performs the following operations:

a. Immediately before the impact of bunch $n$, gets from LS-DYNA the density map induced by the impact of all previous bunches.

b. Updates the regions of the FLUKA model which underwent significant density changes.

c. Runs a new FLUKA calculation to be imported in LS-DYNA simulating the impact of bunch $n$.

Results show that the density variation leads to some reduction of deposited energy at each bunch: as shown in Fig. 5, the energy deposition peak is penetrating deeper and deeper at each successive bunch.

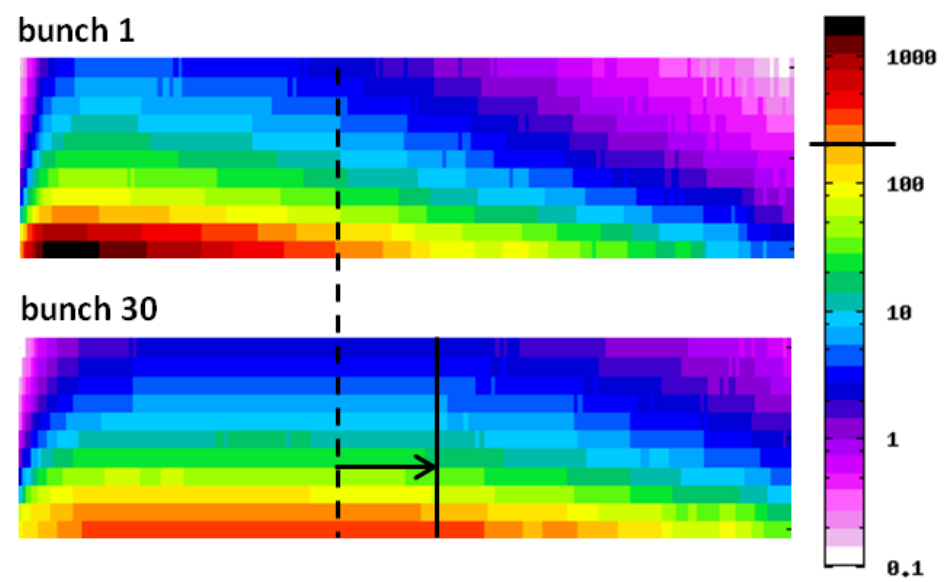

Figure 5. Energy deposition $(\mathrm{GeV} / \mathrm{cm} 3)$ in longitudinal section calculated by FLUKA for $1^{\text {st }}$ and $30^{\text {th }}$ bunches.

Comparison with the uncoupled solution shows that pressure is also affected: its maximum value decreases as the shock wave penetrates into the material, in the beam axis direction. Results also confirm that the differences between coupled and uncoupled analyses are significant only when a substantial density reduction occurs: for the studied cases more than 8 bunches are necessary. 

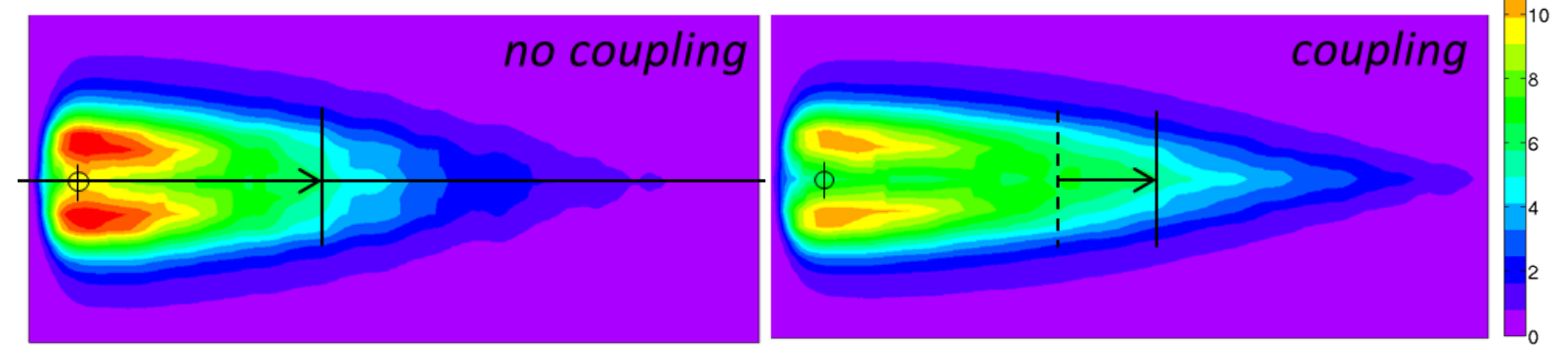

Figure 6. Differences in pressure between Fluka/Hydrocode coupled and uncoupled simulations for a tungsten target impacted by 30 LHC bunches. Pressures are in Pa.

\section{The HRMT-14 Experiment at CERN HiRadMat Facility}

\subsection{Introduction to the experiment}

As shown above, hydrocodes are powerful tools which allow treating extremely complex dynamic phenomena, but require as well, to provide reliable results, constitutive models sufficiently accurate for all the conditions materials may undergo during such events.

In order to gather experimental data for a comprehensive characterization of relevant materials, a specific test (HRMT-14) was performed in October 2012 in the HiRadMat facility. The experimental setup consisted of a multi-material sample holder allowing to test specimens of six different materials impacted by proton beams of different intensity, at the energy of $440 \mathrm{GeV}$.

The specimens were designed in order to generate relatively simple stress waves, with mainly radial and axial components, propagating from the centre of the specimen. These waves were to be measured on the outer face of the specimens by acquiring in real time axial and hoop strains and radial velocity.

Methods traditionally used to experimentally derive EOS rely on the generation of plane waves (Flyer plate, Hopkinson's bar, Taylor's test) in uniaxial strain regime, relatively simple to interpret and study [32].

However, when energy is rapidly deposited inside solids, as it is the case for the interaction of intense particle beams with matter, the propagating stress waves cannot be treated with the uniaxial strain assumption. The method adopted to validate or define equations of state is hence rather different: intensities, shapes and frequencies of stress waves measured on outer surfaces of specimens are benchmarked against those anticipated by numerical simulations. In case of good agreement between simulations and experiment, one may reasonably conclude that the constitutive models, essentially derived from theoretical investigations, are acceptable, at least from an integral point of view.

On top of strain gauges, optimized to collect data at very high sampling rates to fit expected shock wave profiles with sufficient accuracy, additional instrumentation was also installed, namely temperature sensors, microphones and vacuum pressure gauges. Projections of particles generated by the beam impact on specimens were filmed by a high-speed camera.

\subsection{Tested Materials}

Three of the tested materials were relatively conventional pure metals or metallic alloys: Inermet ${ }^{\circledR}$ 180, Glidcop ${ }^{\circledR}$ AL-15 and Molybdenum. Three novel composites, currently under intense development at CERN, were also tested, namely Molybdenum-Copper-Diamond (MoCuCD), Copper-Diamond (CuCD) and Molybdenum-Graphite(-Carbon Fibers) (MoGR) [31]. 


\subsubsection{Inermet ${ }^{\circledR} 180$}

Inermet ${ }^{\circledR} 180$ is a commercial tungsten heavy alloy which combines excellent thermal and mechanical properties with high density.

The material is obtained by liquid-phase sintering of a powder mixture composed of tungsten $(95 \%)$, nickel $(3.5 \%)$ and copper $(1.5 \%) ; 100 \mu \mathrm{m}$-large tungsten grains are immersed in the low-melting $\mathrm{Ni}-\mathrm{Cu}$ phase which provides thermal and electrical continuity. Besides, nickel enhances the diffusion of tungsten atoms through the grain borders improving particle adhesion, thus increasing mechanical properties [33].
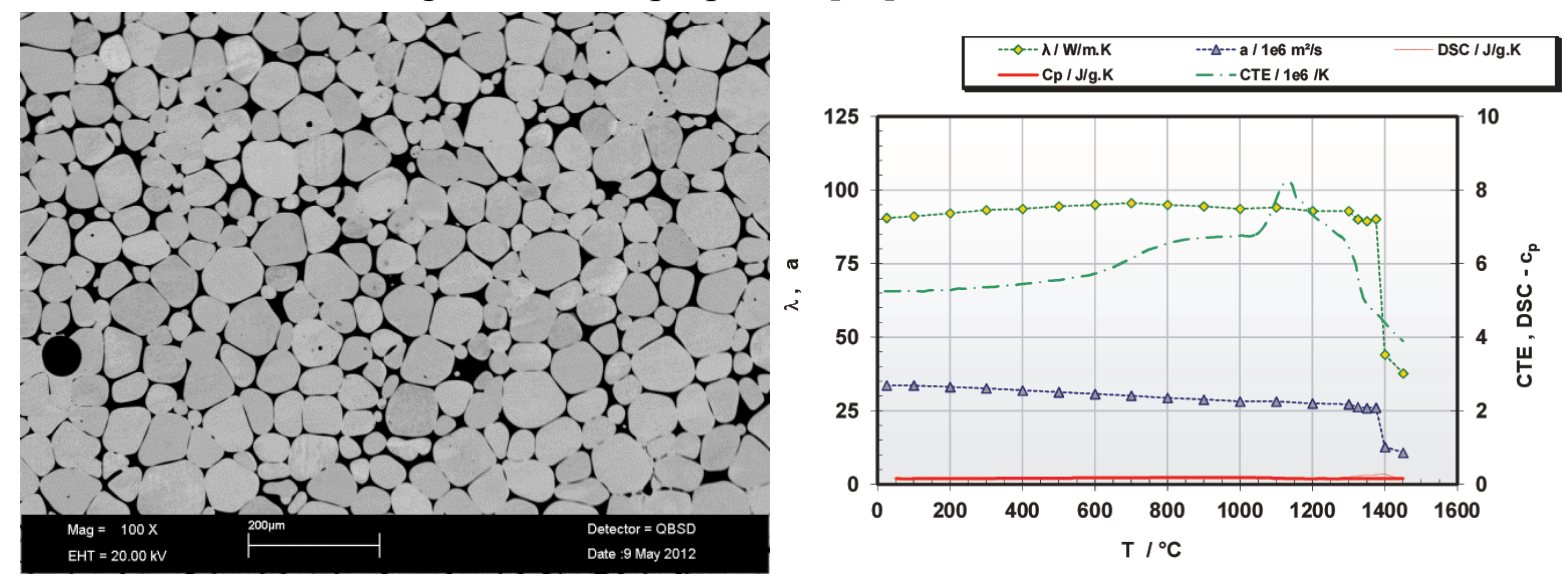

Figure 7. SEM observations with backscattered electron detector of Inermet ${ }^{\circledR} 180$ microstructure (left); B) thermal properties measured up to $1450^{\circ} \mathrm{C}$ (right).

\subsubsection{Molybdenum}

Molybdenum is produced by sintering pure powders at temperatures over $2000{ }^{\circ} \mathrm{C}$; the material is later cold-worked to achieve $50 \%$ reduction in thickness. In the lamination direction, the fracture elongation can thus reach up to $40 \%$. The microstructure is made of stretched Mo grains with small inclusions, mostly of $\mathrm{Mo}_{2} \mathrm{C}$, finely dispersed into the matrix (Fig. 8).

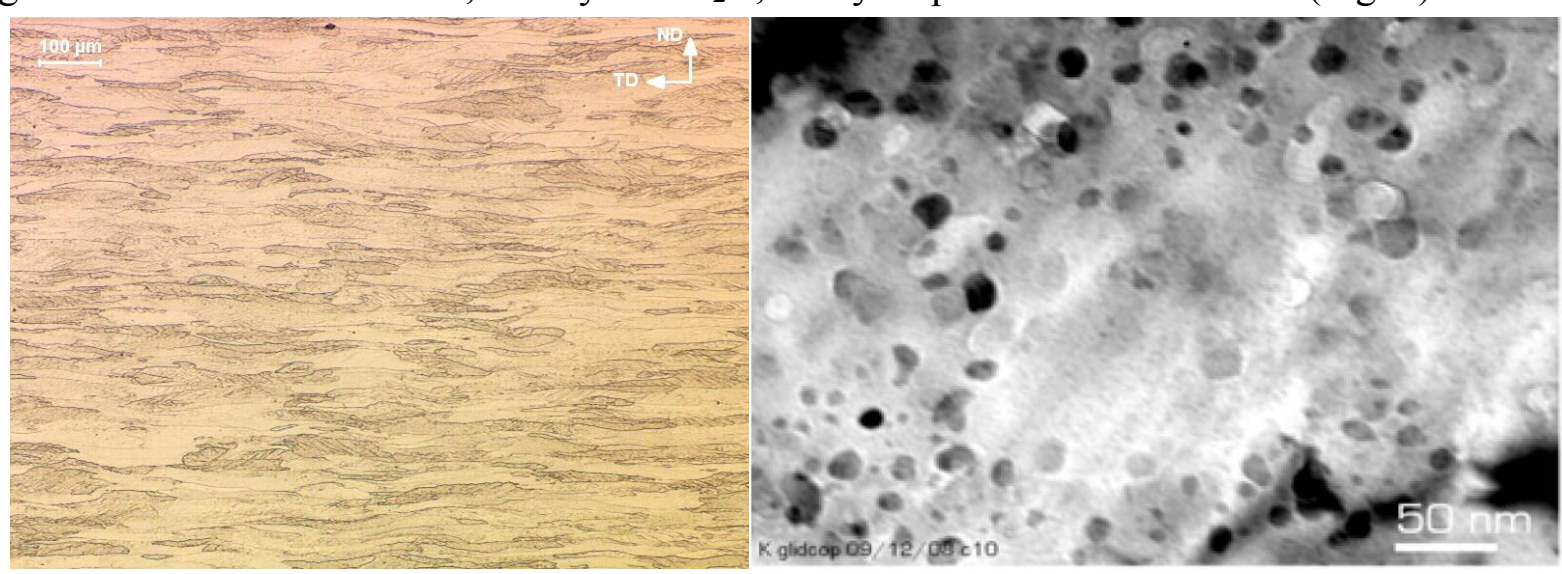

Figure 8. Optical microscope observation of cold worked molybdenum: inclusions are visible as black spots in the molybdenum matrix (left); Glidcop ${ }^{\circledR}$ AL-15 microstructure features small alumina particles dispersed into the copper matrix (right).

\subsubsection{Glidcop ${ }^{\circledR}$ AL-15}

Glidcop ${ }^{\circledR}$ AL-15 is an alumina-strengthened copper produced from copper grains with a small percentage of aluminium, mixed with copper oxide powders. The solid-state reaction generates alumina particles (Fig. 8), finely dispersed into the matrix, which reduce dislocation movements and grain growth at high temperatures. As a result, the material keeps good 
mechanical strength at high temperatures, while maintaining thermo-physical properties comparable to those of pure copper.

\subsubsection{Molybdenum-Copper-Diamond (MoCuCD)}

Molybdenum-Copper-Diamond is a novel composite material jointly developed by CERN and an Italian SME, BrevettiBizz (Verona): goal of the R\&D program is to combine the mechanical strength and electrical conductivity of metals with the high thermal properties, low CTE and low density of diamond.

The material is obtained by liquid-phase sintering of small synthetic diamonds $(40 \%)$, pure molybdenum (35\%) and pure copper powders $(25 \%)$ through rapid hot-pressing. During the sintering process at $1200^{\circ} \mathrm{C}$, molybdenum forms stable carbides on the surface of diamonds, improving mechanical adhesion of the elements; on the other hand, the temperature is not high enough to completely sinter molybdenum grains: the addition of a low-melting filler increases the compaction rate (Fig. 9). Copper also enhances thermal and electrical properties.

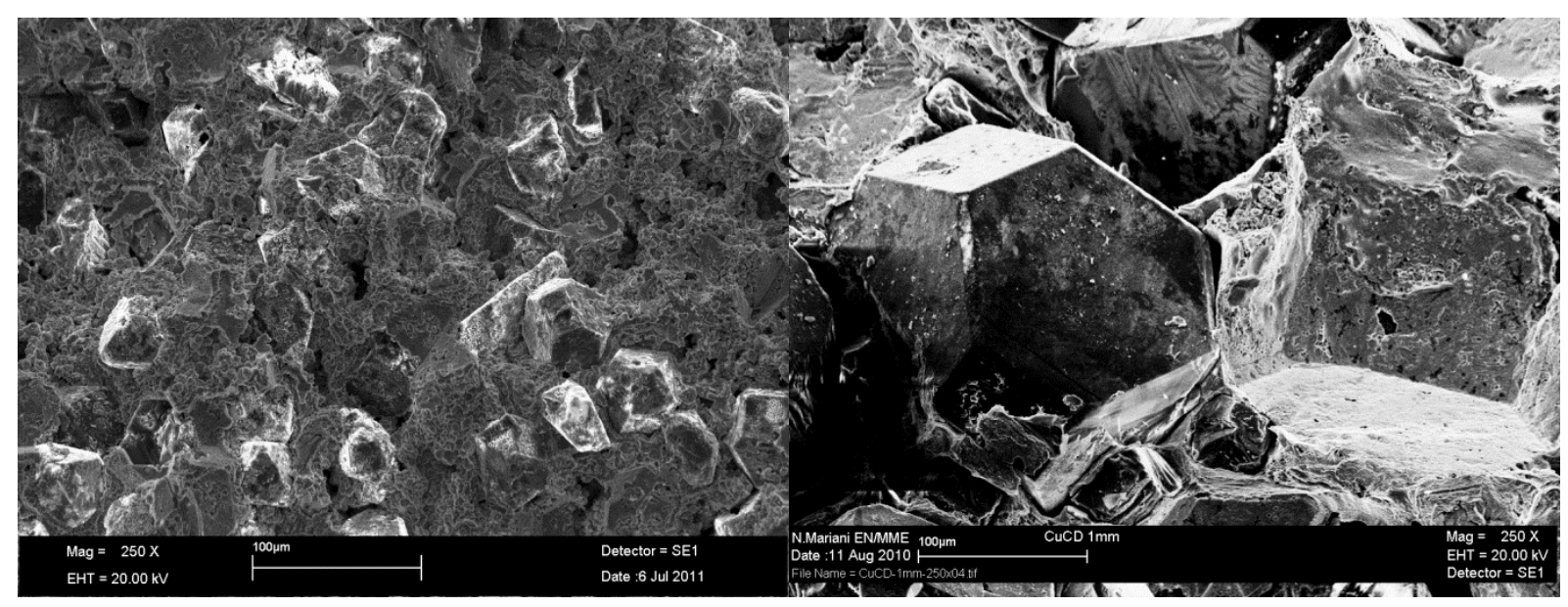

Figure 9. SEM view of MoCuCD microstructure: $45 \mu \mathrm{m}$-large diamonds in the Mo-Cu matrix (left); SEM view of CuCD: $175 \mu \mathrm{m}$ diamonds surrounded by the $\mathrm{Cu}$ phase. The white spots on diamond surfaces are boron carbides (right).

\subsubsection{Copper-Diamond (CuCD)}

Copper-Diamond (CuCD) has been developed by AIT (Austrian Institute of Technology, Seibersdorf, A), now RHP-Technology, and studied for particle accelerator applications in the frame of the EuCard collaboration.

$\mathrm{CuCD}$ is made of $60 \%$ synthetic diamonds, $39 \%$ copper powder and $1 \%$ boron powder, mixed and sintered by rapid hot pressing: diamonds enhance the material thermal conductivity while decreasing density. Boron forms carbides at diamond interfaces and slightly dissolves in copper, assuring mechanical bonds between the two main phases. This interface has however inherent limitations due to the brittleness of boron carbides and to its reduced extent.

\subsubsection{Molybdenum-Graphite composites (MoGR)}

The presence of low-melting phases inside MoCuCD and CuCD strongly affects the resistance of these materials in case of beam impacts [34]. To overcome this limitation, a new family of composites in under development at CERN and BrevettiBizz: Molybdenum-Graphite (MoGR).

The preferential recrystallization of $\{1000\}$ graphite planes during rapid hot pressing at temperatures up to $2200^{\circ} \mathrm{C}$ leads to a heavily anisotropic structure, assuring high thermal 
properties in the principal direction and fair mechanical properties. The high mobility of carbon atoms favours a solid-state reaction between molybdenum and graphite, which generate $\mathrm{Mo}_{2} \mathrm{C}$; a secondary $\mathrm{MoC}$ phase, finely dispersed inside the primary carbide phase, is also formed. Fig. 10 (left) shows recrystallized graphite grains (black), $\mathrm{Mo}_{2} \mathrm{C}$ primary phase (light grey) and $\mathrm{MoC}$ inclusions (black acicular bands inside the $\mathrm{Mo}_{2} \mathrm{C}$ grains).

In order to further increase the thermal properties of MoGR, the addition of carbon fibres is currently under development; first tests show that thermal conductivity is substantially increased, although fibres tend to lower the compaction rate, somehow reducing mechanical strength. Further developments are therefore aiming at minimizing material porosities.

MoGR specimens, both with and without carbon fibres, were tested during the HRMT-14 experiment.

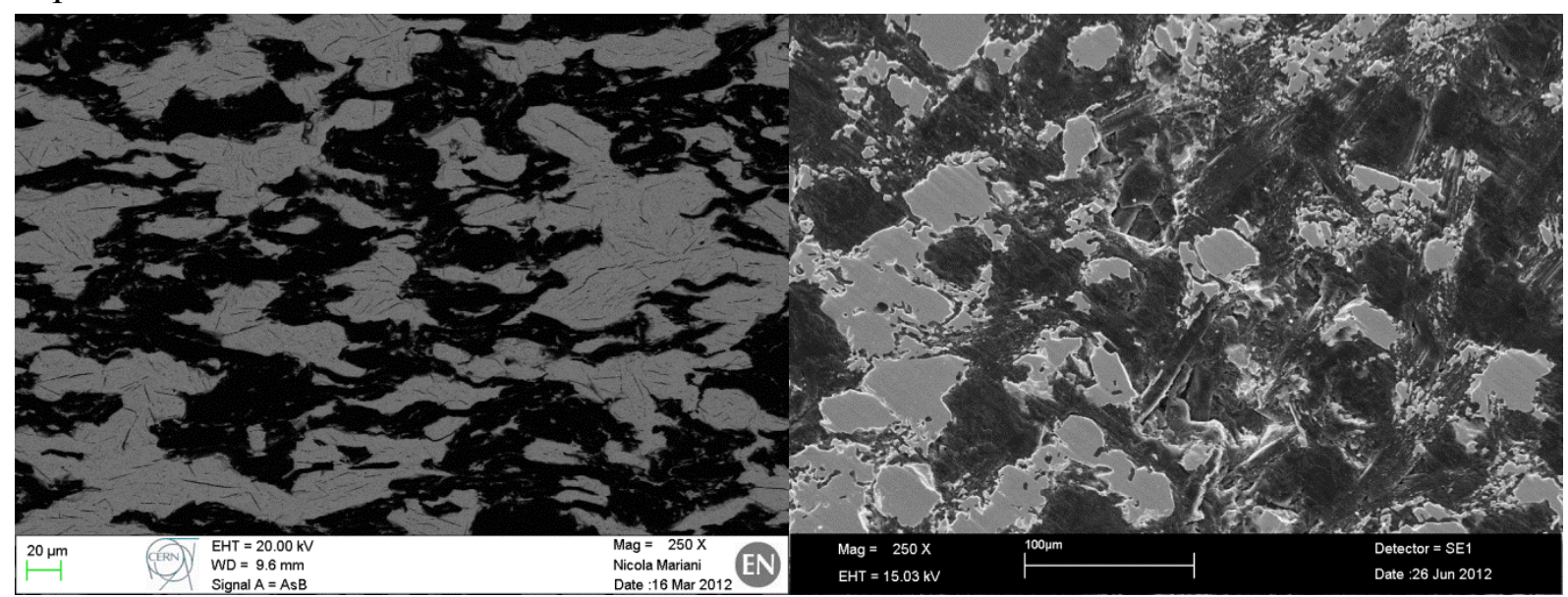

Figure 10. MoGR without carbon fibres (left); MoGR with carbon fibres: porosities are clearly visible (right).

\subsection{Test-bench layout}

The test-bench was primarily constituted by a vacuum vessel and a specimen housing featuring 12 material sample tiers arranged in two rows of six (Fig. 11).

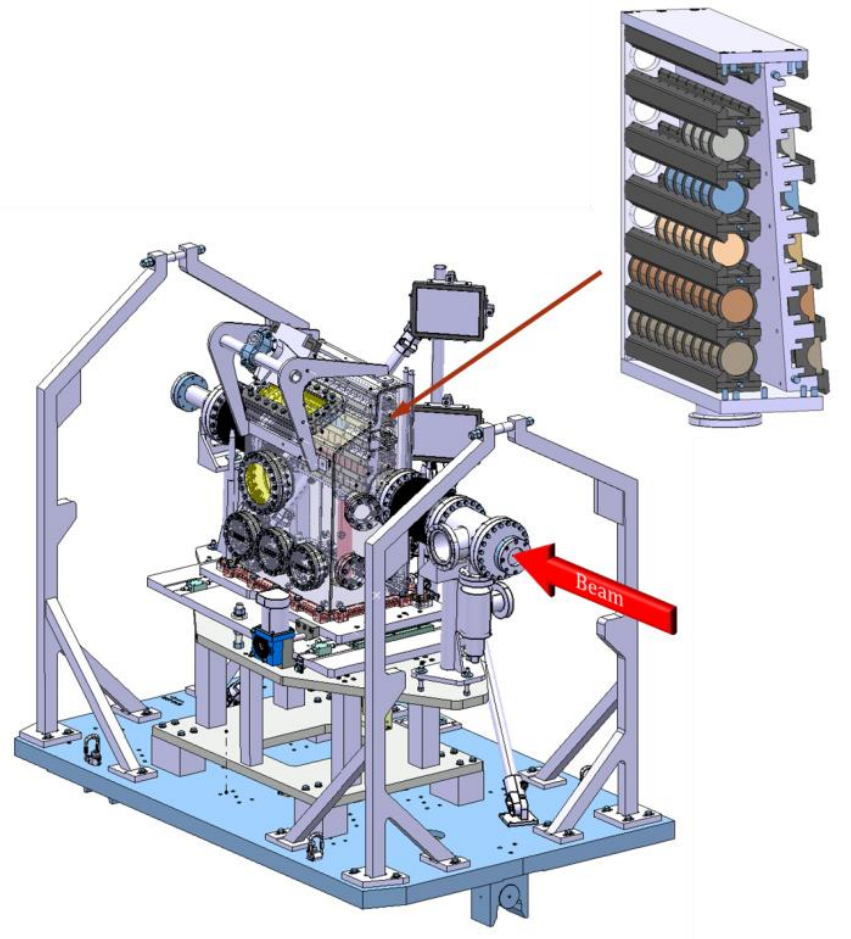

Figure 11. General assembly of the HRMT-14 test-bench. 
The specimen housing could be accurately positioned via a two degrees-of-freedom actuation system. The $300-\mathrm{mm}$ vertical travel permitted to align with the beam axis each of the six tiers, while the 120-mm lateral movement permitted to switch between the two arrays.

Two different specimen shapes were chosen for each material to be tested: cylindrical disks (type 1) for medium intensity tests, to measure axially symmetric shock waves; cylinders with a half-moon cross section (type 2) for high intensity tests, allowing extreme surface phenomena (melting, material explosion, debris projections etc.) to be visualized and optically acquired. The diameter of both sample types is $40 \mathrm{~mm}$, their length $30 \mathrm{~mm}$. The flat surface of type 2 is $2 \mathrm{~mm}$ from the centre of the cylinder.
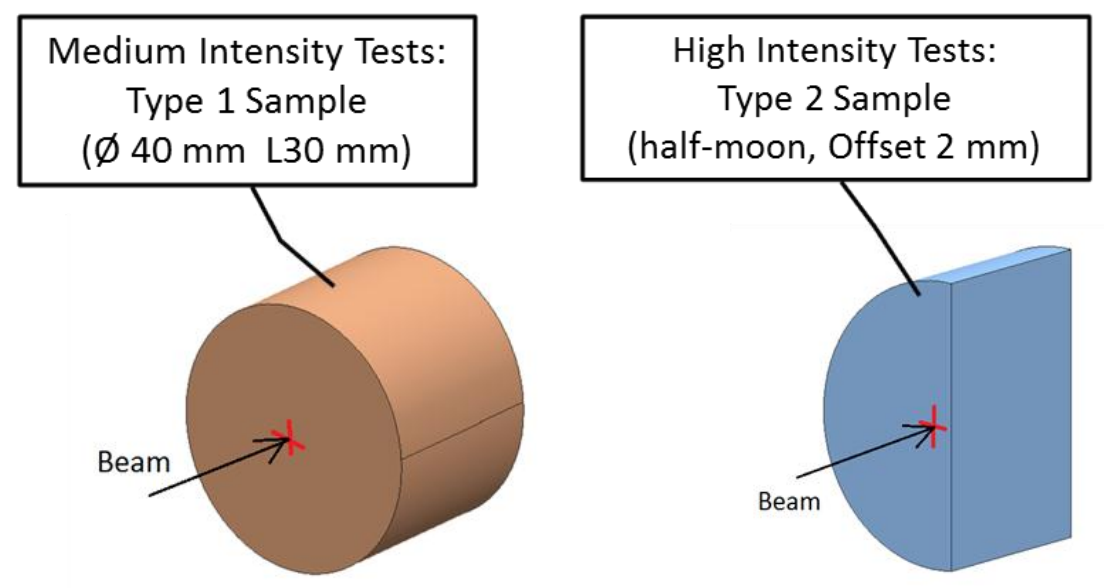

Figure 12. Material specimen shapes for medium intensity (type 1 - left) and high intensity (type 2 - right).

Material specimens were kept in place by graphite restraints to minimize the propagation of shock waves into the housing (thanks to shock impedances mismatch). The number of specimens per tier varied as a function of the radiation and nuclear interaction lengths of the sample material.

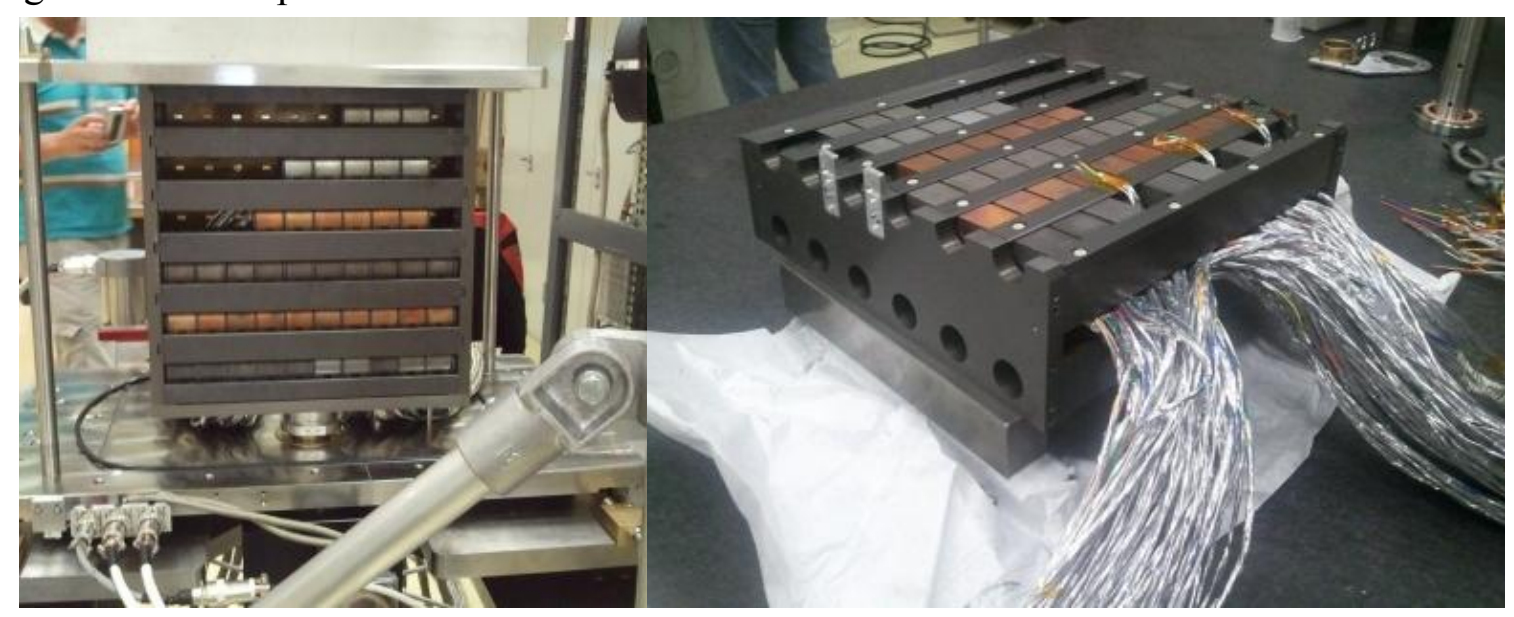

Figure 13. Material sample holder: type 1 samples side (left) and type 2 side (right).

The stainless-steel-made vacuum vessel hosted a series of view ports on its sides to allow online measurements and offline observations. Each of these ports housed an optically transparent window, designed to withstand internal vacuum and fragment impacts.

One view port allowed the transmission of a laser beam for laser Doppler vibrometer (LDV) measurements on type 1 specimens (lateral window). A second port was dedicated to the image acquisition of type 2 samples while exposed to high intensity shots. The vessel was equipped with UHV beryllium windows at the beam entry and exit ports. 


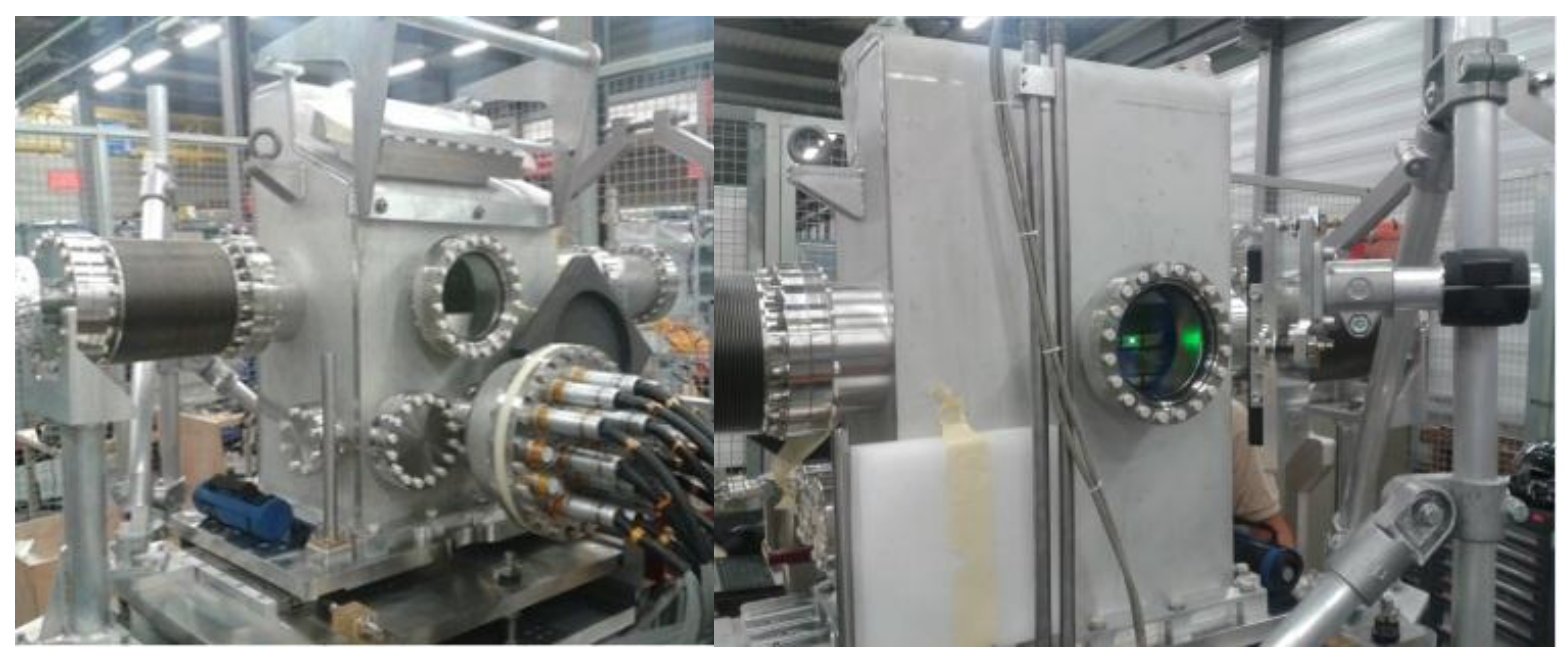

Figure 14. Assembled test-bench with DAQ cables and connectors (left); viewport for LDV measurements (right).

Medium vacuum ( $\leq 1 \mathrm{mbar}$ ) was required for the experiment. After installation in the tunnel, a pump placed on the table maintained suitable vacuum level, even after outgassing provoked by beam impacts.

\subsection{Experiment digital acquisition system}

Embedded and remote data acquisition systems were characterized and installed; during test runs, all measurement devices were synchronized by a machine (accelerator) trigger.

The whole instrumentation was designed in order to capture, in amplitude and frequency, the signals expected after beam impacts. Extensive simulations were performed during the design phase on materials for which more reliable constitutive models were available (Inermet and Glidcop) to specify DAQ requirements; Fig. 15 shows an example of the expected hoop strain on an Inermet specimen. Table 1 provides the main features of the DAQ system.
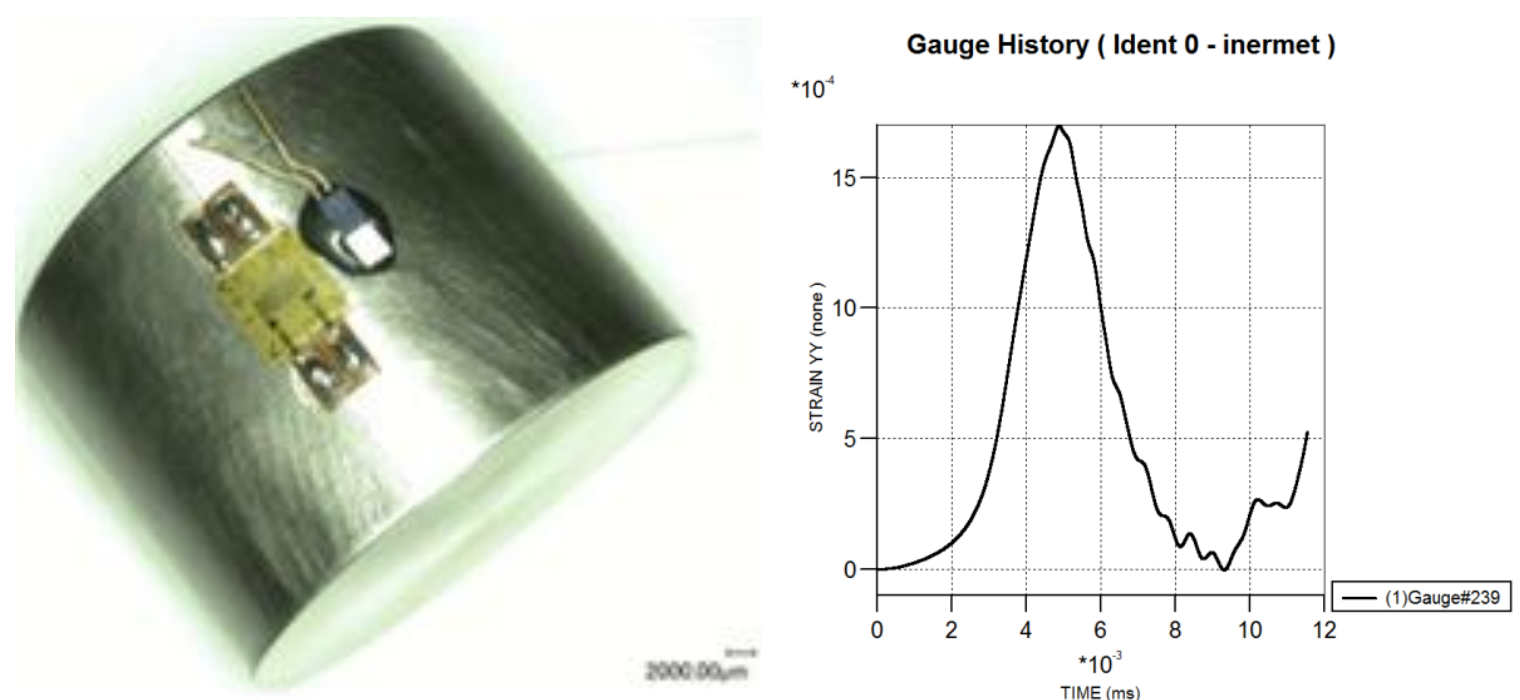

Figure 15. Equipped Inermet specimen showing PT100 and bidirectional strain gauge (left); expected hoop strain on the centre of an Inermet specimen, energy $440 \mathrm{GeV}$, 3e12 protons/pulse (right). 
Table 1: Main characteristics of the DAQ system

\begin{tabular}{|c|c|c|c|c|c|}
\hline & Type of measurement & $\begin{array}{l}\text { Acquisition } \\
\text { range }\end{array}$ & Quantity & Sensor type & $\begin{array}{l}\text { Sampling } \\
\text { frequency }\end{array}$ \\
\hline \multirow{3}{*}{$\begin{array}{l}\text { Electrical } \\
\text { Systems }\end{array}$} & Surface Strain & $100 \div 5000 \mu \mathrm{m} / \mathrm{m}$ & 244 & $\begin{array}{c}\text { Strain } \\
\text { gauges }\end{array}$ & $4 \mathrm{MHz}$ \\
\hline & Temperature & $-150 \div 150^{\circ} \mathrm{C}$ & 36 & PT100 & $100 \mathrm{~Hz}$ \\
\hline & Vacuum & $\begin{array}{c}\sim 10^{-4} \div 1000 \\
\text { mbar }\end{array}$ & 1 & Pirani gauge & $100 \mathrm{~Hz}$ \\
\hline \multirow{2}{*}{$\begin{array}{l}\text { Optical } \\
\text { Systems }\end{array}$} & Radial velocity & $<0.1 \div 24 \mathrm{~m} / \mathrm{s}$ & 1 & LDV & $4 \mathrm{MHz}$ \\
\hline & $\begin{array}{l}\text { Particle front } \\
\text { propagation }\end{array}$ & $0 \div 500 \mathrm{~m} / \mathrm{s}$ & 1 & $\begin{array}{l}\text { High-speed } \\
\text { camera }\end{array}$ & $20000 \mathrm{fps}$ \\
\hline
\end{tabular}

Strains reaching out sample surface were measured via resistive strain gauges glued on the cylindrical face of both type 1 and type 2 samples: both azimuthal and longitudinal components were acquired. The temperature response of each material after the impact was monitored by PT100 thermal probes; a total of 244 strain gauges and 36 thermal sensors were installed.

Given the high radiation level expected during the experiment, only radiation-hard equipment could be installed in-situ. All embedded components (strain gauges, thermal sensors, adhesives, connectors and cables) had a resistance before failure higher than $250 \mathrm{kGy}$ (10 times the expected prompt dose) [35].

Table 2 shows the resistance to radiation of each element: suggested operating ranges and failure prompt doses are reported [36],[37],[38],[39].

Table 2: Radiation resistance of embedded DAQ components

\begin{tabular}{|c|c|c|}
\hline Material & Operating range & Failure \\
\hline Strain gauge & $2 \cdot 10^{8} \mathrm{~Gy}$ & $6 \cdot 10^{8} \mathrm{~Gy}$ \\
\hline Adhesive & $\sim 10^{5} \mathrm{~Gy}$ & $10^{8} \mathrm{~Gy}$ \\
\hline Solder & Not available & $2 \cdot 10^{7} \mathrm{~Gy}$ \\
\hline Cables & $2.5 \cdot 10^{5} \mathrm{~Gy}$ & $10^{8} \mathrm{~Gy}$ \\
\hline Connectors & $\sim 10^{5} \mathrm{~Gy}$ & $10^{7} \mathrm{~Gy}$ \\
\hline
\end{tabular}

Due to limitations as to the number of available cables in the HiRadMat facility, a radiation-hard multiplexing hardware was developed (8:1 $\mathrm{In} /$ Out ratio) and installed on the testbench. Multiplexer control was transferred to the surface control room, since underground facilities were not accessible during the experiment.

In order to validate the sampling frequency and maximum amplitude of the strain gauges, extensive tests were performed with a Hopkinson bar setup at Politecnico di Torino to reproduce the stresses and strains expected on the specimens.

Optical devices (laser Doppler vibrometer and high-speed camera) were remotely installed in order to protect them from the effects of radiations.

The LDV acquired the radial velocity on the outer surface of one cylindrical sample per tier. Thanks to the axial symmetry of the layout, radial velocities could be handily converted in hoop strains, after time integration, so to cross-check strain gauge measurements. 
A high-speed camera was used to film the particle projection produced by impacts on type 2 specimens; the lighting necessary for the acquisition was provided by a battery of radiation-hard xenon flashes mounted atop the tank.

A system of precisely aligned mirrors was put in place to reflect the laser beam and the beam impact images back to the vibrometer and to the high speed camera, positioned inside a shielded bunker, $40 \mathrm{~m}$ upstream of the experimental area.

\subsection{Preliminary results}

A very large amount of data was acquired during the experiment and is currently under intense post-processing. As said above, the main goals of the experiment were to corroborate the simulation methods, increasing confidence in advanced simulation techniques, validate existing material constitutive models and derive new constitutive models for less known materials. This is mainly done by benchmarking strains and velocities measured on the outer surface of the specimens as well as pictures taken during impacts on type 2 samples against simulation results and by post-irradiation analyses of impacted specimens.

Records concerning better known materials (Inermet and Glidcop) were treated first.

Experimental data and numerical simulations were compared for type 1 Glidcop specimens. Table 3 summarizes most relevant parameters for experiment and simulations.

Table 3: Beam and material parameters, medium intensity tests on Glidcop

\begin{tabular}{|l|c|c|}
\hline & Experiment parameters & Simulations parameters \\
\hline Specimen material & Glidcop AL-15 & Glidcop AL-15 / OFE Copper \\
\hline Proton Energy & $440 \mathrm{GeV}$ & $440 \mathrm{GeV}$ \\
\hline Number of bunches & 72 & 72 \\
\hline Pulse intensity & $4.66 \mathrm{e} 12$ protons & $4.66 \mathrm{e} 12$ protons \\
\hline Bunch spacing & $25 \mathrm{~ns}$ & $25 \mathrm{~ns}$ \\
\hline Impact point & Centre of the specimen & Centre of the specimen \\
\hline $\begin{array}{l}\text { Beam Transverse dimension } \\
\text { (Standard Deviation) }\end{array}$ & $1.3 \times 1.3 \mathrm{~mm}^{2}$ & $2.5 \times 2.5$ mm² \\
\hline EOS & - & Copper OFE - SESAME table \\
\hline Strength model & - & Glidcop AL-15 - Johnson-Cook \\
\hline Failure model & - & Copper OFE - Johnson-Cook \\
\hline
\end{tabular}

Since for simulations, only Glidcop strength model was available [39], the EOS and failure model of pure copper were adopted instead. In spite of this approximation and of the difference in beam transverse size, experimental measurements (strain gauges and LDV) and simulations are in very good agreement. In Fig. 16, red, black and green dotted lines are referred to the hoop strains measured by strain gauges placed on the external surface with $90^{\circ}$ azimuthal spacing: in principle, given the axial symmetry of load and geometry, the three lines should be superposed. Differences in measured values must be analysed, but are possibly mainly due to noise on cables and gauges during the acquisition as well as to errors on the beam impacting position. It is also interesting to note that electromagnetic noise induced by the particle beam perturbed the strain gauge measurements during a few microseconds after the impact, concealing the first deformation peak; however, this interference died out immediately after, allowing to capture the remainder of the phenomenon. 

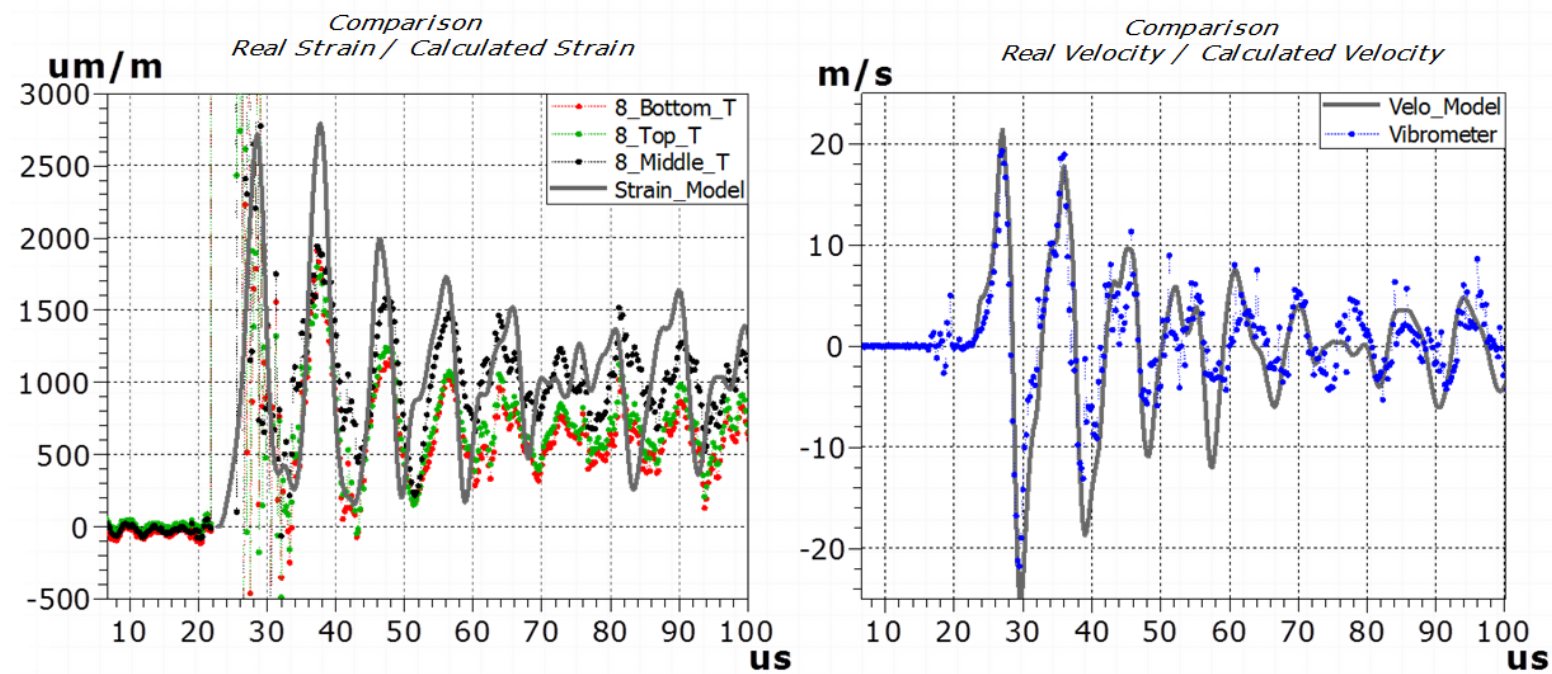

Figure 16. Hoop strain: comparison between measurements of three strain gauges and numerical model (left); radial velocity measured by the vibrometer, in blue, and simulated one, in black (right).

The high-speed camera and flash systems allowed for the first time, to the best of authors' knowledge, to record images of the impact of a hadron beam on solid targets and of the effects this induced. The most remarkable phenomena occurred during beam impact on type 2 specimens made of Inermet, the material with the highest stopping power.

Table 4 provides impact parameters for experiment and simulations.

Table 4: Beam and material parameters, high intensity tests on Inermet

\begin{tabular}{|c|c|c|}
\hline & Experiment parameters & Simulations parameters \\
\hline Specimen material & Inermet@ 180 & - \\
\hline Proton Energy & $440 \mathrm{GeV}$ & $440 \mathrm{GeV}$ \\
\hline N. bunches & 72 & 60 \\
\hline Pulse intensity & $9.05 \mathrm{e} 12$ protons & $9 \mathrm{e} 12$ protons \\
\hline Bunch spacing & $25 \mathrm{~ns}$ & $25 \mathrm{~ns}$ \\
\hline Observed sample & $2^{\text {nd }}$ & $3^{\text {rd }}$ \\
\hline Impact point & $2 \mathrm{~mm}$ from the flat surface & $2 \mathrm{~mm}$ from the flat surface \\
\hline $\begin{array}{l}\text { Beam Transverse dimension } \\
\text { (Standard Deviation) }\end{array}$ & $1.9 \times 1.9 \mathrm{~mm}^{2}$ & $2.5 \times 2.5 \mathrm{~mm}^{2}$ \\
\hline EOS & - & Tungsten - SESAME table \\
\hline Strength model & - & Tungsten Johnson-Cook \\
\hline Failure model & - & Tungsten Hydro (Pmin) \\
\hline
\end{tabular}

As shown in Figs. 17-18, a large quantity of hot material was ejected at high velocity from the two most loaded Inermet samples; the high temperatures reached are confirmed by the intense light emitted by the fragments during a few hundred microseconds. 

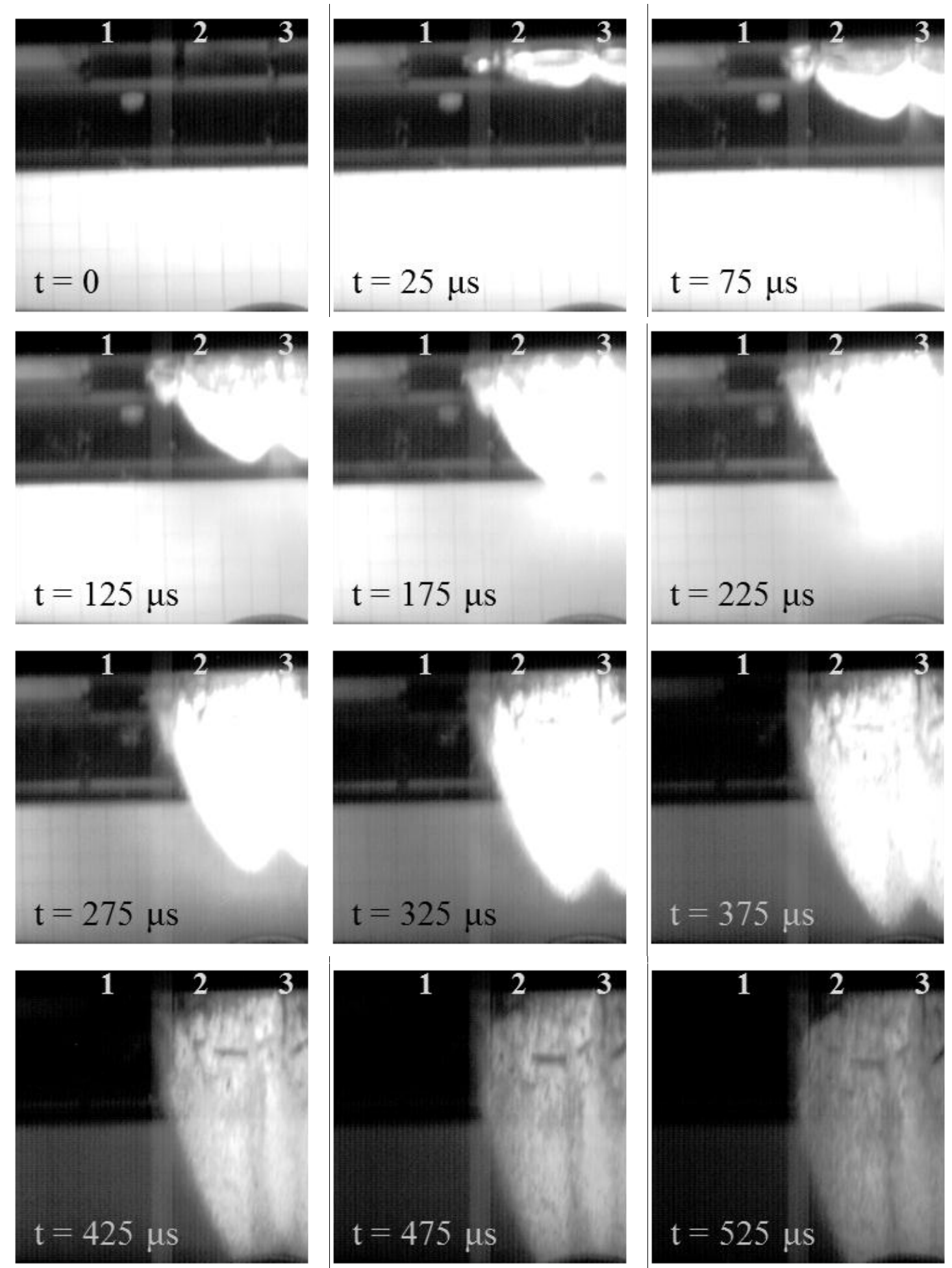

Figure 17. Image sequence of the impact on Inermet 180 of a 72 bunches proton pulse. Beam is coming from the left; three Inermet samples are partially visible (numbered 1 to 3 ). 


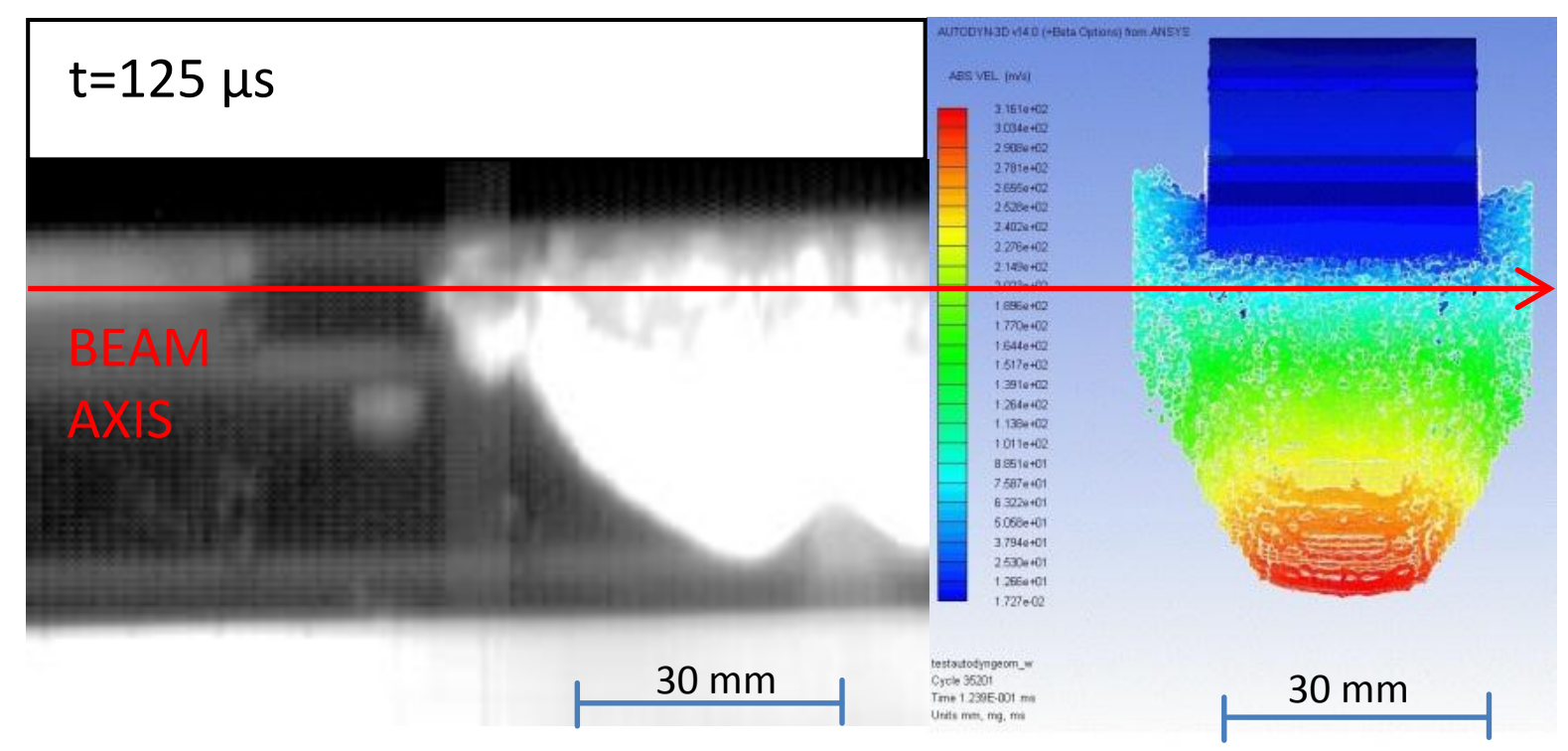

Figure 18. Comparison between simulation (SPH method) and acquired image $\sim 125 \mu$ s after the impact.

Both ejected particle front shape and velocity are consistent with high-speed camera acquired data (Fig. 18), even considering the differences between real and the simulated scenarios.

The estimated velocity of the fragment front, as acquired, is $\sim 275 \mathrm{~m} / \mathrm{s}$, well matching the simulated velocity of $316 \mathrm{~m} / \mathrm{s}$.

\subsection{Discussion of preliminary results and future steps}

The good fitting between numerical results and experimental measurements confirms the reliability of the simulation techniques and provides a positive indication on the validity of the EOS and strength model for Glidcop. The matching between captured pictures of Inermet explosion and SPH simulations goes in the same direction. Similar analyses will be performed in the near future on molybdenum for which constitutive models are existing (although less well established than for copper and tungsten).

However, additional sensitivity analyses are necessary to assess the influence of the extreme conditions reached at and around the most impacted areas of materials on the propagation of stress waves in the less loaded regions: this would be particularly important to ascertain if large fluctuations in the constitutive models at the most extreme conditions (for which data are less reliable) can be correctly inferred on the outer surface of the samples or if these tend to die out.

As said above, for novel materials the approach will be somehow different: combining measurements and theoretical work, constitutive models will be conjectured and implemented in the hydrocode models, then the comparison of simulations results with measured values should allow to iteratively converge towards consistent material models.

\subsection{Post-irradiation: initial observations}

Only limited and rapid visual observations were allowed after the experiment on account of the high residual dose levels. Valuable information was nevertheless gathered about the beam impact resistance of the densest materials (Fig. 19). 


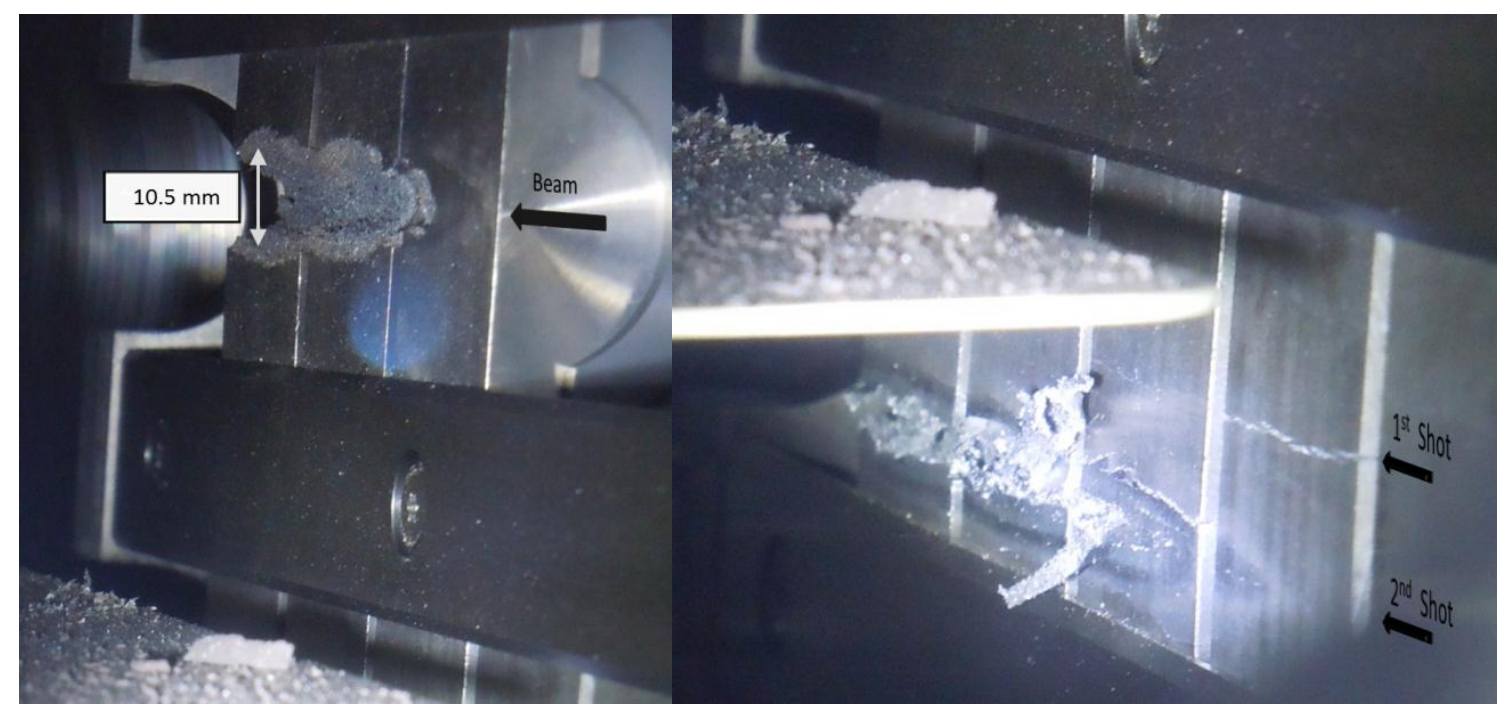

Figure 19. Damage on Inermet provoked by impact of 72 bunches, $9.05 \mathrm{e} 12$ protons, beam transverse standard deviation $(\sigma) 1.9 \mathrm{~mm}$ (left); thermo-mechanical effects produced on molybdenum by two pulses at different vertical positions: 72 bunches, $9.05 \mathrm{e} 12$ protons and 144 bunches, $1.96 \mathrm{e} 13$ protons, $\sigma 1.9 \mathrm{~mm}$.

Inermet experienced a brittle failure, with no signs of plastic deformation on the brim of the damaged area and on the flat surface. The low-melting point of copper and nickel probably played an important role in determining the extent of damaged zone.

The simulated damage extension is consistent with experimental observations (Fig. 20).

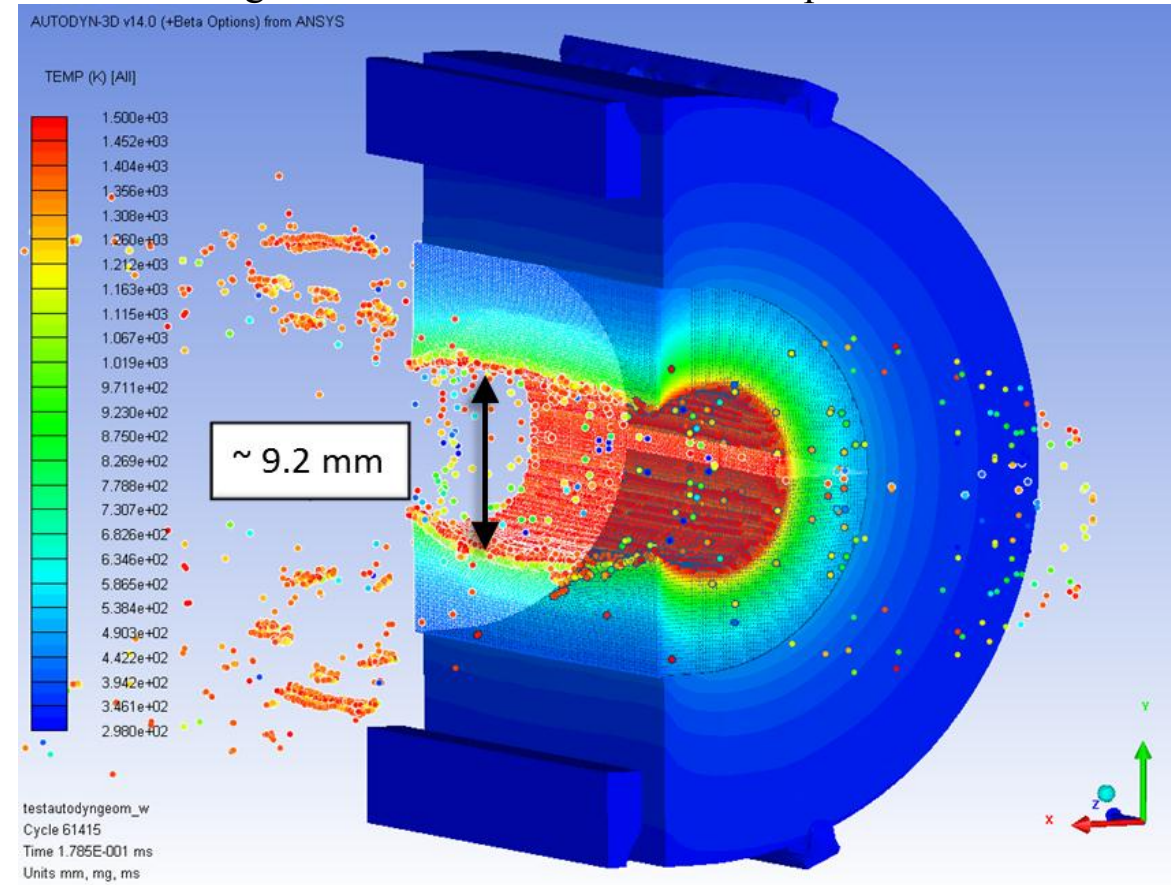

Figure 20. Simulated damage extension on Inermet specimen. Simulation parameters: 72 bunches, $1.08 \mathrm{e} 13$ protons, beam transverse standard deviation $2.5 \mathrm{~mm}$.

It is interesting to note that, for the same energy and intensity, no apparent damage can be observed on the surface of molybdenum specimens, if we except a surface crack generated on a less loaded specimen, for which metallographic investigations must be performed, once the tank can be opened. A second pulse was shot on the same samples at double intensity with a vertical offset: a groove was this time produced on the surface of most loaded specimens; even 
so, its extension was still lower than in the Inermet case; Fig. 19 also evidences a ductile fracture, with plastic deformation confirmed by metallic burrs still attached to the samples.

Extensive post-irradiation analyses are to take place in future months once the set-up has reached a manageable level of activation. The post-irradiation campaign will be carried out in an adequately equipped laboratory and include visual observations, direct measurements, nondestructive and destructive tests as well as metallurgical observations of relevant specimens. This will contribute to complete material data which have been collected during the simulation and experimental phases.

\section{Conclusions}

A complex and comprehensive experiment has recently been carried out at CERN to assess the consequences of highly energetic particle pulses impacting beam intercepting devices: this is a fundamental issue in state-of-the-art facilities developed for high-energy particle physics.

The experiment aimed at the characterization, mostly in real time, of six different materials impacted by $440 \mathrm{GeV}$ intense proton pulses. Chosen materials were a combination of relatively conventional metals and of novel composites, currently under development. The design of the test set-up required innovative solutions in terms of lighting, support stabilization, radiation resistance and noise control.

The test was a success under any point of view: all experiment systems (DAQ, electronics, and mechanics) worked properly in spite of the very harsh environment and of the technological challenges. The high-speed camera and flash systems allowed for the first time, to the best of authors' knowledge, to record images of the impact of a hadron beam on solid targets and of the effects this induced.

Preliminary measurements on Glidcop and Inermet specimens very well match results of advanced computations, confirming the reliability of the simulation methods and supplying promising indications on the validity of constitutive models used for these materials.

A very large amount of data is ready to be treated to help deriving constitutive models for the less known materials, namely for the novel composite material which were tested for the first time during the experiment.

An extensive post-irradiation campaign, implying direct observations, non-destructive and destructive testing, is to be launched in future months to complete the experiment and provide additional valuable information.

The experimental method presented in this paper may find applications to test materials under very high strain rates and temperatures in domains well beyond particle physics (severe accidents in fusion and fission nuclear facilities, space debris impacts, fast and intense loadings on materials and structures etc.), particularly when little explored radial shock waves are generated.

\section{References}

[1] The LHC Design Report, http://ab-div.web.cern.ch/ab-div/Publications/LHCDesignReport.html, July 2004.

[2] R. Schmidt et al., New Journal of Physics, 8 (2006) 290.

[3] A. Bertarelli et al., "The Mechanical Design for the LHC Collimators", EPAC'04, Lucerne, July 2004.

[4] R. Assmann et al. "Requirements for LHC Collimation System", EPAC'02, Paris, June 2002.

[5] S. Peggs, “The European Spallation Source”, PAC'11, New York, March 2011. 
[6] A. Bertarelli, T. Kurtyka, "Dynamic thermo-mechanical phenomena induced in isotropic cylinders impacted by high energy particle beams", $8^{\text {th }}$ International Conference on Structures Under Shock and Impact, Crete, Greece, 29 - 31 Mar 2004.

[7] A. Dallocchio, "Study of Thermo-Mechanical Effects Induced in Solids by High Energy Particle Beams: Analytical and Numerical Methods", PhD Thesis, Politecnico di Torino, Turin 2008.

[8] A. Bertarelli et al., "Limits for Beam Induced Damage: Reckless or Too Cautious?", Proc. Chamonix 2011: Workshop on LHC Performance, January 2011.

[9] A. Bertarelli et al., "Mechanical Design for Robustness of the LHC Collimators", PAC'05, Knoxville, May 2005.

[10] A. Bertarelli, A. Dallocchio, T. Kurtyka, J. Appl. Mech. - ASME, 75 (3) (2008) 1-13.

[11] A. Bertarelli et al. "Permanent Deformation of the LHC Collimator Jaws Induced by Shock Beam Impact: an Analytical and Numerical Interpretation", EPAC'06, Edinburgh, July 2006.

[12] J.A. Zukas, "Introduction to Hydrocodes", Elsevier, Amsterdam, 2004.

[13] J.H. Tillotson, "Metallic Equations of State for Hypervelocity Impact", Report No. GA-3216, July 18 (General Atomic, San Diego, California, 1962).

[14] K. S. Holian (Ed.), LANL report LA-10160-MS, 1984.

[15] G. R. Johnson, W. H. Cook, "A Constitutive Model and Data for Metals Subjected to Large Strains, High Strain Rates and High Temperatures", Proc. 7th Int. Symp. on Ballistics, 1983.

[16] D. J. Steinberg, S. G. Cochran, M. W. Guinan, J. Appl. Phys. 51 (3) (1980) 1498-1504

[17] G. R. Johnson and T. J. Holmquist, "An Improved Computational Constitutive Model for Brittle Materials" pp. 981-4 in High Pressure Science and Technology, 1993, Vol. 2, Edited by S. C.Schmidt, J. W.Shaner, G. A.Samara, and M.Ross. AIP Press, New York, 1994.

[18] D. E. Grady, J. Mech. Phys. Solids, 36 (3) (1988) 353-384.

[19] “ANSYS Autodyn User Manual, Release 13.0”, ANSYS Inc., 2010.

[20] B. Gladman et al., "LS-DYNA® Keyword User's Manual - vol. I - Version 971", LSTC, 2007.

[21] European Coordination for Accelerator Research \& Development http://eucard.web.cern.ch/eucard/index.html.

[22] G. Battistoni et al., "The FLUKA code: Description and Benchmarking", Proc. of the Hadronic Shower Simulation Workshop 2006, Fermilab, AIP Conference Proceeding, 2007.

[23] A. Fassò, A. Ferrari, J. Ranft, and P.R. Sala, "FLUKA: a Multi-particle Transport Code" CERN-2005-10 (2005), INFN/TC_05/11, SLAC-R-773.

[24] A. Bertarelli et al., "High Energy Beam Impacts on Beam Intercepting Devices: Advanced Numerical Methods and Experimental Set-Up", IPAC'11, San Sebastián, September 2011.

[25] J.J. Monaghan, Computer Physics Communications, 48 (1988) 88-96.

[26] G. Ben-Dor, O. Igra, T. Elperin (Eds.), "Handbook of Shock Waves, volume 1: Theoretical, Experimental and Numerical Techniques", Academic Press, 2001, pp. 327328.

[27] I. Efthymiopoulos et al., "HiRadMat: a New Irradiation Facility for Material Testing at CERN", IPAC'11, San Sebastián, September 2011.

[28] M. Cauchi et al., "High Energy Beam Impact Tests on an LHC TCT Collimator at CERN HiRadMat Facility", submitted to IPAC'13, Shanghai, May 2013.

[29] M. Scapin, L. Peroni, A. Dallocchio, J. Nucl. Mater. 420 (2012).

[30] M. Scapin, L. Peroni, V. Boccone, F. Cerutti, "A LS-DYNA/FLUKA coupling for the numerical simulation of high energy particle beam interaction with matter", submitted 
to $5^{\text {th }}$ Conference on Computational Methods for Coupled Problems in Science and Engineering, Ibiza, June 2013.

[31] A. Bertarelli et al., "Research and Development of Novel Advanced Materials for Next-generation Collimators", IPAC'11, San Sebastián, September 2011.

[32] M. A. Meyers, "Dynamic Behavior of Materials", Wiley-Interscience, New York, 1994

[33] C.W. Corti, Platinum Metals Rev., 30 (4) (1986).

[34] M. Scapin, L. Peroni, A. Bertarelli, A. Dallocchio, N. Mariani, S. Bizzaro, "Investigation of the behavior of Metal-Diamond Composites", Presentation at ECCM15, June 2012, Venice, Italy.

[35] N. Charitonidis et al., "Radiological assessment of the Collimator Materials tests at HiRadMat in 2012", EPFL-Report-178219.

[36] H. Schonbacher, M. Tavlet, "Compilation of radiation damage test data, Part I", CERN, 1989.

[37] F. Guarino, C. Hauviller, M. Tavlet, "Compilation of radiation damage test data, Part IV", CERN, 2001.

[38] K. Hoffmann, "An Introduction to Measurements using Strain Gauges", Hottinger Baldwin Messtechnik Gmbh, Erkrath, Germany, 1989.

[39] G. Lee, IEEE T. Nucl. Sci., NS-32 (5) (1985).

[40] M. Scapin, L. Peroni, M. Peroni, Int. J. Impact Eng., 41-42 (2011). 